\title{
Wnt-induced Vangl2 phosphorylation is dose-dependently required for planar cell polarity in mammalian development
}

\author{
Wei Yang ${ }^{1}$, Lisa Garrett ${ }^{2}$, Di Feng ${ }^{1}$, Gene Elliott ${ }^{2}$, Xilin Liu $^{2,3}$, Ni Wang ${ }^{1}$, Yu Ming Wong ${ }^{1}$, Nga Ting Choi ${ }^{1}$, \\ Yingzi Yang ${ }^{2,4}$, Bo Gao ${ }^{1,2,5}$

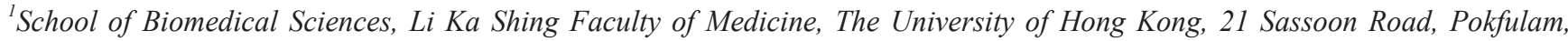 \\ Hong Kong, China; ${ }^{2}$ National Human Genome Research Institute, National Institutes of Health, Bethesda, MD 20892, USA; ${ }^{3}$ Chi- \\ na-Japan Union Hospital of Jilin University, Changchun, Jilin 130033, China; ${ }^{4}$ Department of Developmental Biology, Harvard \\ School of Dental Medicine, 188 Longwood Ave., Boston, MA 02115, USA, ${ }^{5}$ Centre for Reproduction, Development and Growth \& \\ HKU-SUSTEC Joint Laboratories of Matrix Biology and Diseases, The University of Hong Kong, Pokfulam, Hong Kong, China
}

Planar cell polarity (PCP) is an evolutionarily conserved essential mechanism that provides directional information to control and coordinate polarized cellular and tissue behavior during embryonic development. Disruption of PCP leads to severe morphological defects in vertebrates and its dysregulation results in a variety of human diseases such as neural tube defects and skeletal dysplasia. PCP is governed by a set of highly conserved core proteins that are asymmetrically localized at the cell surface throughout the polarized tissues. The uniform directionality of PCP is established by global cues, such as $\mathrm{Wg} / \mathrm{Wnt}$ signaling gradients that break the original symmetrical localization of core PCP proteins including Vang/Vangl and Fz/Fzd. However, the exact mechanism remains elusive. In this study, we found that Vangl2 phosphorylation, which was previously identified to be induced by Wnt5a signaling, is required for Vangl2 functions in mammalian PCP in multiple tissues. The in vivo activities of Vangl2 are determined by its phosphorylation level. Phospho-mutant Vangl2 exhibits dominant negative effects, whereas Vangl2 with reduced phosphorylation is hypomorphic. We show that Vangl2 phosphorylation is essential for its uniform polarization pat-

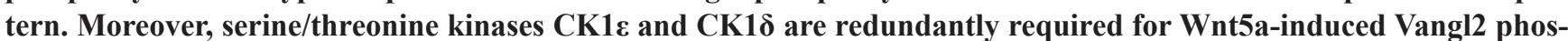
phorylation. Dvl family members are also required for Wnt5a-induced Vangl2 phosphorylation by enhancing the interaction of CK1 and Vang12. These findings demonstrate that induction of Vangl protein phosphorylation plays an essential role in transducing Wnt5a signaling to establish PCP in mammalian development, suggesting a phosphorylation-regulated "Vangl activity gradient" model in addition to the well-documented "Fz activity gradient" model in Wnt/PCP signaling.

Keywords: Wnt; planar cell polarity; PCP; Vang12; mammalian development

Cell Research (2017) 27:1466-1484. doi:10.1038/cr.2017.127; published online 20 October 2017

\section{Introduction}

Planar cell polarity (PCP) originally refers to the polarization of epithelial cells within a plane orthogonal to their apical-basal axis and has been further extended

Correspondence: Yingzi Yang ${ }^{\mathrm{a}}, \mathrm{Bo} \mathrm{Gao}^{\mathrm{b}}$

${ }^{a}$ Tel: +1-617-432-8304; Fax: +1-617-432-3246

E-mail: yingzi yang@hsdm.harvard.edu

${ }^{\mathrm{b}}$ Tel: +852 3917-6809; Fax: +852 2855-1254

E-mail: gaobo@hku.hk

Received 30 October 2016; revised 16 May 2017; accepted 20 August 2017; published online 20 October 2017 to mesenchymal cells. The establishment of PCP is a critical, evolutionarily conserved process during development and organogenesis [1-5]. Genetic studies of PCP in Drosophila identified a key conserved "core" pathway composed of a group of core proteins, including Frizzled (Fz), Dishevelled (Dsh), Diego (Dgo), Flamingo (Fmi), Prickle (Pk), and a four-pass transmembrane protein Van Gogh (Vang) [6-17]. PCP is evolutionarily conserved from Drosophila to mammals. While the similarities between Drosophila and vertebrate PCP establishment are overwhelming, analyses in several vertebrate model organisms, such as zebrafish, Хenopus, and mouse, have identified novel regulatory factors and regulatory 
schemes in the regulation of PCP and have also defined new cellular features requiring PCP signaling input. In mammals, multiple homologs of the core PCP proteins exist. For example, Drosophila Vang has two homologs in mammals, namely, Vang-like 1 (Vangl1) and Vanglike 2 (Vangl2). Vangll and Vangl2 are redundantly required for PCP and they together regulate mammalian morphogenesis in a dose-dependent manner $[18,19]$. Similarly, mammalian homologs of Drosophila Dsh, $D v l 1, D v l 2$, and $D v l 3$ are also redundantly required for PCP [20-22]. In addition to the redundancy, mammals also require additional PCP components, such as Ror2, Ryk, and PTK7. Consequently, novel regulatory schemes have been developed to control mammalian PCP [23-26]. In mammals, PCP controls a diverse array of cellular, developmental, and physiological processes, such as directed cell migration, left-right patterning, arrangement of inner ear sensory hair cells, skeletal morphogenesis, axon guidance, and epidermal wound healing [1-4, 19, 27-33]. Dysregulation of PCP signaling in humans is associated with neural tube defects, neurological disorders (e.g., epilepsy), skeletal defects, and other human diseases, including cancer [24, 34-41]. For example, mutations in human ROR2, its ligand WNT5A, as well as DVL1 and $D V L 3$ cause a severe skeletal dysplasia, Robinow syndrome [42-46].

During the establishment of PCP, core PCP proteins, which are originally randomly distributed, become asymmetrically localized $[6,16,47-50]$. This asymmetry is uniform throughout the planar polarized tissue and is a molecular hallmark of PCP, which is followed by uniform polarized cellular behavior, such as coordinated cell orientation and biased localization of hair or cilia [4, $5,51,52]$. Several symmetry-breaking cues, including Wingless (Wg)/Wnt signaling, the Fat-Dachsous-Fourjointed system, and mechanical tension imposed by tissue morphogenesis, have been proposed to establish such uniform directionality [53-61]. Among them, Wg/Wnt secreted molecules have long been hypothesized to provide global instructive cues largely because of their graded expression pattern and their receptors $\mathrm{Fz} / \mathrm{Fzd}$ are being core PCP proteins [49, 53-55, 62-64]. A study in Drosophila found that $\mathrm{Wg}$ and $\mathrm{dWnt} 4$ provide global cues to orient PCP by forming gradients. These gradients could possibly generate an $\mathrm{Fz}$ activity gradient by modulating the Fz-Vang interaction across the cell membrane [65]. In other model systems, Wnt proteins were also found to orient PCP [66-68]. In addition to the Fz activity gradient model $[49,55]$, we have previously proposed a Vangl activity gradient model for mouse limb morphogenesis [24]. We found that Wnt5a induces Vang12 phosphorylation in two clusters of highly conserved serines (Ser) and thre- onines (Thr) through a Wnt5a receptor Ror2 in a Wnt5a dose-dependent manner [24]. More Ser and Thr residues are induced to undergo phosphorylation as a result of increased Wnt5a doses. This suggests that the Wnt5a dose is transduced intracellularly by different levels of Vangl2 phosphorylation. In cultured mammalian cells, Vangl2 phosphorylation occurs in a relay such that phosphorylation on three priming sites (S84 and S82 in cluster I and S5 in cluster II, Supplementary information, Figure S1) is required for other sites to be phosphorylated [24]. Vangl2 phosphorylation is functionally important in vertebrate PCP during zebrafish and Xenopus gastrulation [24, 69]. A very recent study also identified two phosphorylation sites, S120 and S122, on Drosophila Vang protein, which are equivalent to the mouse Vang12 priming sites S82 and S84 (Supplementary information, Figure S1). While it is unknown whether Wg or dWnt4 could regulate Vang phosphorylation, loss of phosphorylation at S120 and S122 led to disruption of Vang asymmetric distribution and loss of PCP in Drosophila [70]. Interestingly, Vang11 and Vangl2 phosphorylation sites were found to be mutated in human patients with neural tube defects [71, 72]. These findings highlighted a potential key role of Vangl2 phosphorylation in establishing PCP, although the genetic evidence in mammals is lacking and the in vivo dose effect of Vangl2 phosphorylation remains to be tested. The Vangl protein "activity" may be quantitatively modulated by its phosphorylation level.

Here, for the first time, we determined the in vivo requirement of Vang12 phosphorylation in mammalian embryonic morphogenesis by analyzing different lines of Vangl2 phospho-mutant mice that we generated. We showed that Vangl2 phosphorylation is critical for its function in PCP. Furthermore, the phosphorylation levels quantitatively regulate Vangl 2 activities in mice. Phospho-mutant Vangl2 exhibits dominant negative effects, whereas Vangl2 with reduced phosphorylation is hypomorphic. We also found important evolutionary differences in the regulation and functional outputs of Vang12 phosphorylation by comparison of Wnt/PCP in Drosophila to mice. Our study shows that Casein kinase 1 and Dvl are important regulators in mediating Wnt-induced Vangl2 phosphorylation.

\section{Results}

Phospho-mutant Vangl2 exhibits dominant negative activities

To understand the molecular mechanism whereby Vangl2 phosphorylation regulates PCP, we first examined the cellular localization of wild type and phospho-mutant Vangl2 proteins (Vangl2-Wt and Vangl2-A, respectively). 
The phospho-mutant Vang12 cannot be phosphorylated because all Ser and Thr phosphorylation sites identified in the two clusters of Vangl2 were mutated to alanines (Ala) [24] (Supplementary information, Figure S1). While wild type Vangl2 was largely present on the cell membrane, levels of phospho-mutant Vang12 were reduced on the membrane and the protein was predominantly intracellular (Figure 1A, 1B and Supplementary information, Figure S2A). Tetraspan membrane proteins such as Vang12, characterized by four hydrophobic transmembrane regions and cytoplasmic end domains, are often shuttled between the membrane and endocytic vesicles [73]. This suggested that cytoplasmic Vang12 could represent either unsorted proteins retained in the Golgi/endoplasmic reticulum (ER) or those endocytosed from cell surface. Staining against Golgi/ER markers did not identify significant co-localization (data not shown), whereas labeling with various markers for endocytic vesicles revealed that Vangl2 could be internalized and degraded through endosomes and lysosomes. Notably, most of the phospho-mutant Vangl2 strongly co-localized with endosome and lysosome markers (Supplementary information, Figure S2B). Moreover, blocking of endocytosis significantly increased their membrane localization (Supplementary information, Figure S2C). These findings suggest that phospho-mutant Vangl2 is not stable on the cell surface and is easily endocytosed and degraded through lysosomes.

Given that Vang11 and Vangl2 can oligomerize [74, 75 ] and that Ror2 and Vangl2 can form a protein complex [24], we examined the effects of phospho-mutant Vangl2 on wild type Vangl and Ror2 proteins following their co-transfection. We found that the membrane localization of wild type Vang11, Vangl2, and Ror2 was significantly disrupted and they became strongly associated with phospho-mutant Vangl2 in cytoplasmic compartments, such as lysosomes (Figure 1A, 1B and Supplementary information, Figure S3A, S3C). This phenomenon indicates a dominant negative effect of phospho-mutant Vangl2 on its binding partners. The dominant negative effects were further supported by the reduced protein level of wild type Vangl and Ror2 proteins when co-expressed with phospho-mutant Vang12 (Supplementary information, Figure S4A). The Vang12 loop-tail mutation (Lp, S464N) also showed a dominant negative effect by which wild type Vangl proteins were retained in the ER [29, 75, 76] (Supplementary information, Figure S3B) although it did not affect Ror2's membrane localization (Supplementary information, Figure S3B, S3C). These results suggested different dominant negative effects conferred by loss of phosphorylation and loop-tail mutation. The Vangl2-Lp mutant blocks the transport of wild type Vangl proteins from the ER to the plasma membrane, whereas the phospho-mutant Vangl2 triggers endocytosis of its binding partners.

To test the functional significance of Vangl2 phosphorylation in vivo, we generated two inducible Vangl2 knock-in mouse lines to express wild type and phospho-mutant Vangl2 in a Cre-dependent manner from the Rosa26 locus [77] (Figure 1C). This approach has been widely used to induce gene expression and compare gene variant functions in mice. These alleles were designated as Rosa-Vangl2 ${ }^{W t}$ (wild type) and Rosa-Vangl2 ${ }^{A}$ (phospho-mutant). A $3 \times$ HA tag was fused to the N-terminal of Vangl2 to enable specific and convenient detection of the Vang12 expressed from the Rosa26 locus (Figure 1C). As Vangl2 is ubiquitously expressed in early embryos [19], the $\beta$-actin-Cre or Sox2-Cre [78, 79] was used to activate global Vangl2 expression in the entire early embryo to test its function. The $\beta$-actin-Cre;Rosa-Vangl2 ${ }^{W t /+}$ and Sox2-Cre;Rosa-Vangl2 ${ }^{W t+}$ embryos and mice were viable and normal (Figure 1D and data not shown), suggesting that the dose of induced wild type Vangl2 expression was within the physiological threshold. By contrast, expression of phospho-mutant Vangl2 in the Rosa-Vangl2 ${ }^{A}$ line resulted in a typical PCP phenotype in mouse, which is an open neural tube defect (Figure 1D). Quantitative real-time PCR analysis revealed that the mRNA level of induced wild type Vangl2 was approximately 50\% that of the endogenous Vangl2 level. Moreover, the mRNA level of induced phospho-mutant Vangl2 was similar to that of the induced wild type Vangl2 (Figure 1E).The amount of induced Vangl 2 proteins (detected by HA antibody) in Rosa-Vangl $2^{A}$ embryos was reduced compared to Rosa-Vangl2 ${ }^{\text {Wt }}$ embryos (Figure 1F) suggesting that phospho-mutant Vangl2 is unstable. The instability of phospho-mutant Vangl2 was confirmed by a cycloheximide chase analysis using mouse embryonic fibroblasts (MEFs) isolated from Rosa-Vangl2 ${ }^{W t}$ and Rosa-Vangl ${ }^{A}$ embryos (Supplementary information, Figure S4B). Notably, total Vangl2 and Vangl1 protein levels were lower in Rosa-Vangl2 $2^{A}$ embryos (Figure 1F), suggesting a dominant negative effect exerted by phospho-mutant Vangl 2 in vivo. However, the open neural tube phenotype of $\beta$-actin-Cre;Rosa-Vangl2 ${ }^{A /+}$ is unlikely to result from an insufficient dose of Vang12 or Vangl1 because the total Vangl2 and Vangl1 protein level was still approximately $80 \%$ of wild type (Figure $1 \mathrm{~F}$ ) and Vangll $^{+/-}$; Vangl2 ${ }^{+/-}$ mice are normal [19].

As a key molecular feature of PCP is the asymmetric distribution of core PCP proteins, we examined wild type and phospho-mutant Vangl2 protein localization in planar polarized tissues such as chondrocytes of the developing limb (Figure 1G) [24, 33]. The HA antibody was used 
A
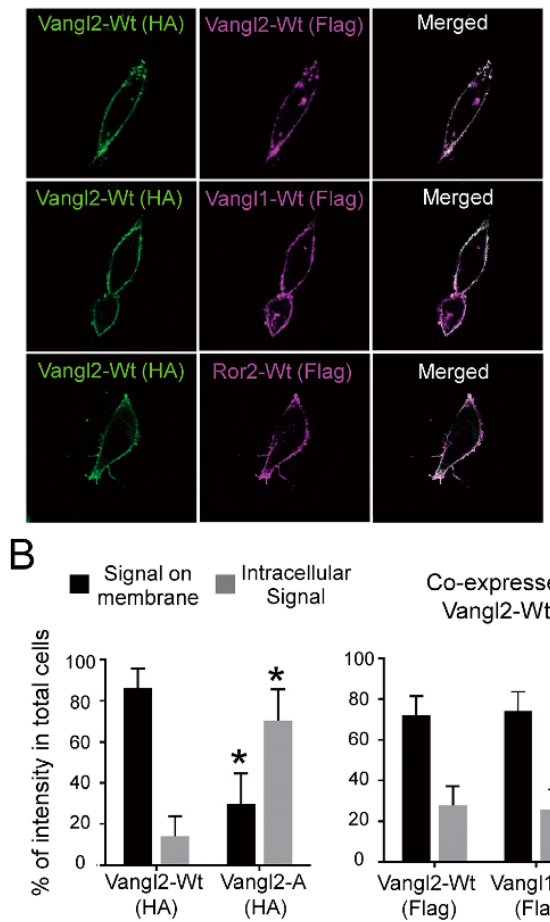

Co-expressed with Vangl2-Wt (HA)

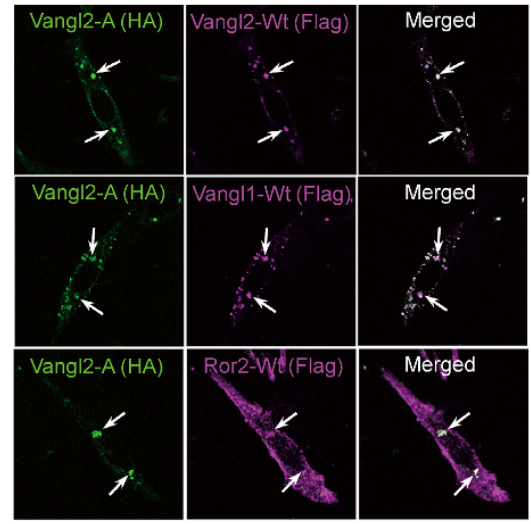

C
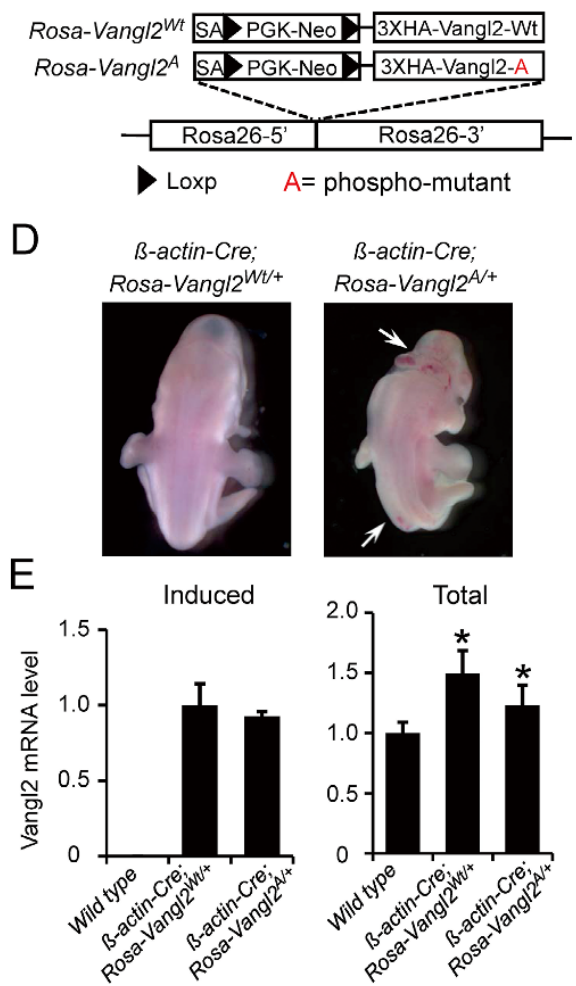

Co-expressed with Vangl2-A (HA)
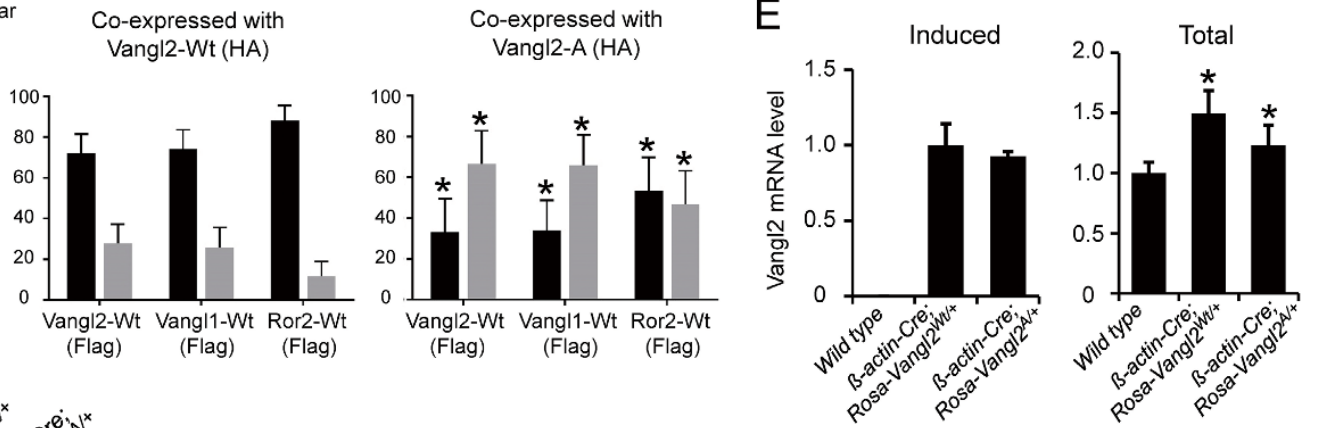

F
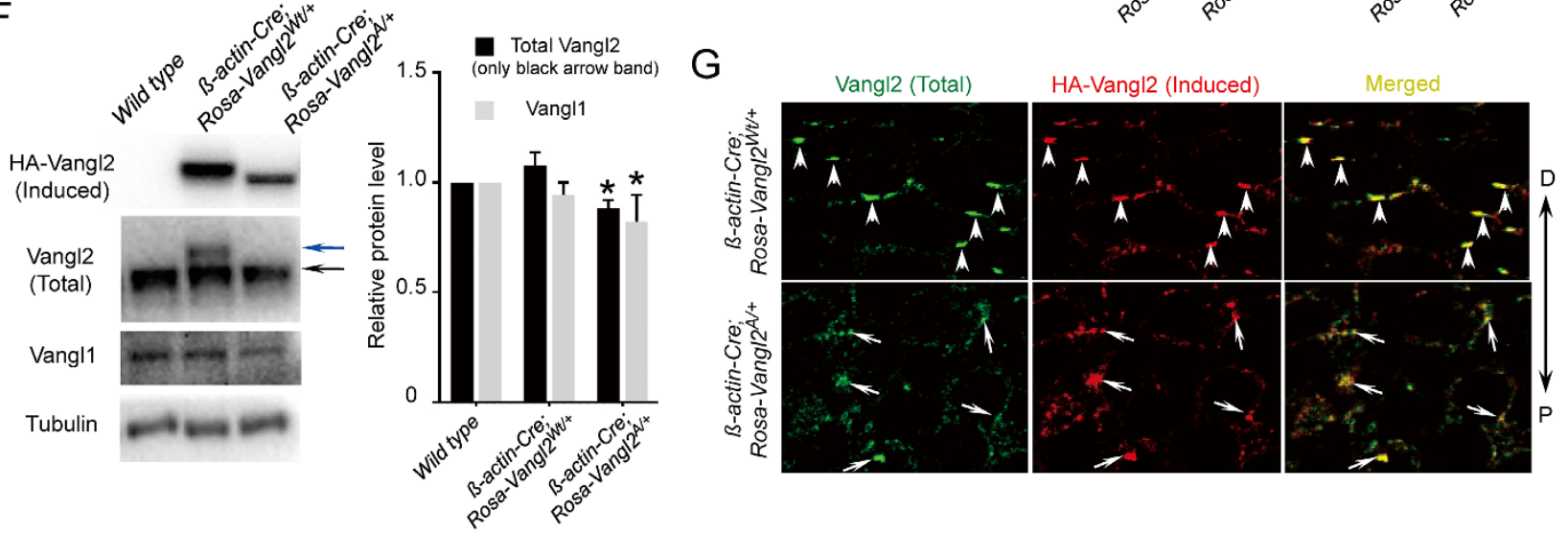

Figure 1 Phospho-mutant Vangl2 exhibits dominant negative effects. (A) Co-localization of HA-tagged Vangl2-Wt (wild type), Vangl2-A (phospho-mutant) with Flag-tagged Vangl2-Wt, Vangl1-Wt or Ror2-Wt in $\mathrm{CHO}$ cells. Arrows point to the intracellular co-localization. (B) The fluorescent signal intensities of different proteins shown in (A) were measured by Image $\mathrm{J}$ and the percentage of the membrane or intracellular signal intensities in total cells was calculated. Number of measured cells: left panel, $n=68,68$; middle panel $n=22,23,23$; and right panel, $n=21,22$, 25. Statistical significance of differences between the groups expressing Vangl2-Wt and Vangl2-A was calculated by two-tailed $t$-test, ${ }^{*} P<0.0001$. Error bars are means \pm SD. (C) Generation of inducible wild type Vangl2 (Rosa-Vang/2 ${ }^{W_{t}}$ ) and phospho-mutant Vang/2 (Rosa-Vangl/24) mouse lines. HAfused mouse Vangl2 cDNA following a floxed PGK-Neo cassette was knocked into the Rosa26 locus. SA, splicing acceptor. (D) $\beta$-actin-Cre-induced Rosa-Vangl2 ${ }^{W t+}$ embryo is normal, but Rosa-Vang/2/2/t embryo shows open neural tube (arrows). (E) Induced and total Vangl2 expression levels in E11.5 wild type, $\beta$-actin-Cre;Rosa-Vang/2 $2^{\text {Wt+ }}$ and $\beta$-actin-Cre;Rosa-Vang/ ${ }^{\alpha++}$ embryos measured by quantitative PCR. GAPDH was used as an internal reference. Two-tailed $t$-test, ${ }^{*} P<0.05$. Error bars are $\pm \mathrm{SD}, n=3$ repetitions. (F) Western blot analysis of E11.5 wild type, $\beta$-actin-Cre;Rosa-Vangl2 ${ }^{\text {Wtt }}$ and $\beta$-actin-Cre;Rosa-Vangl/2 ${ }^{A+t}$ embryos. Quantification of Vangl1 and Vangl2 (bands indicated by black arrow); protein level normalized to tubulin is shown in the right panel $\left(n=3\right.$ repetitions, two-tailed $t$-test, $\left.{ }^{*} P<0.05\right)$. The blue arrow indicates the induced wild type HA-Vangl2. Note that the phospho-mutant HA-Vangl2 and the endogenous phosphorylated wild type Vangl2 have similar molecular weight (lane 3). (G) The localization pattern of induced HA-Vangl2 (red, HA antibody) and total Vangl2 (green, Vangl2 antibody) in E13.5 distal limb chondrocytes of $\beta$-actin-Cre;Rosa-Vangl ${ }^{W t+}$ and $\beta$-actin-Cre;Rosa-Vangl $2^{\alpha+}$ embryos. Arrows point to some of the co-localized signals. P-D, proximal-distal axis of the limb. 
to detect induced Vang12 protein expression from the Rosa26 locus, while Vang12 antibodies were used to detect both endogenous and induced Vangl2 protein expression. First, we found that in the $\beta$-actin-Cre;Rosa-Van$g l 2^{W t /+}$ mice, the induced wild type Vangl2 protein had an identical pattern as that of the wild type endogenous Vang12 (upper row, Figure 1G). By contrast, when the phospho-mutant HA-Vangl2 was induced from the same Rosa26 locus, it failed to exhibit polarized localization and instead was distributed in small puncta. Interestingly, co-localization of induced phospho-mutant HA-Vangl2 with endogenous wild type Vang12 was also detected (lower row, Figure 1G), indicating that phospho-mutant Vangl2 exerts dominant negative effects by hijacking the wild type endogenous Vangl2 protein. In $\operatorname{Vangl} 2^{L p /+}$ mice, wild type Vangl2 proteins could still reach the cell membrane and polarize normally, in spite of the dominant negative effect of Vangl2-Lp (Supplementary information, Figure S3D). This is probably due to the quick degradation of Vangl2-Lp proteins through proteasome (Supplementary information, Figure S4A) [80]. Thus, its dominant negative effect on wild type Vangl proteins is limited.

Phosphorylation of Vangl2 is required for its function in mammalian PCP

To further test the in vivo function of Vangl2 phosphorylation, both Rosa-Vangl2 ${ }^{W t}$ and Rosa-Vangl2 ${ }^{A}$ lines were crossed with the Vangl1 and Vangl2 null mutants. Because of redundant functions of Vangll and Vangl2, Vangl1 ${ }^{-/-}$;Vangl2 ${ }^{+/-}$(designated as Vangl $^{\text {low }}$ ) exhibited an open neural tube in most embryos, while Vangl1 $^{-/}$ or Vangl2 $2^{+-}$embryos were morphologically normal [19]. Rosa-Vangl2 $2^{\text {Wt }}$ completely rescued the phenotypes of the $\mathrm{Vangl}^{\text {low }}$ embryos, whereas phospho-mutant Ro$s a$-Vangl $2^{A}$ could not (Figure 2A, 2B and Supplementary information, Table S1). Interestingly, Rosa-Vangl2 ${ }^{\text {Wt }}$ was unable to rescue other $\mathrm{Vangl}$ mutants with reduced Vangl activities, including the $\operatorname{Vangl1}^{+/+} ; \mathrm{Vangl2}^{-/-}$, Van$\mathrm{gll}^{+/-}$;Vangl2 ${ }^{-/}$, or $\mathrm{Vangll}^{-/-}$;Vangl2 ${ }^{-/-}$mutants (Supplementary information, Table S1). This is because Vangl2 is genetically more potent than Vangl1 [19] and the level of induced Vangl2 protein is limited to less than $50 \%$ of the endogenous level (see middle lane of total Vangl2 in Figure $1 \mathrm{~F}$, where upper band is induced HA-tagged Van$\mathrm{gl} 2$ and the lower band is endogenous Vangl2).

In the inner ear cochlea of the E18.5 mouse embryo, sensory hair cells are organized into four rows (three outer layers and one inner layer). In the Vangl $^{\text {low }}$ mutant, an additional row of sensory hair cells and misoriented hair cells were observed in the first outer layer (Figure 2C). Induced wild type Vangl2, but not phospho-mutant Van- $g l 2$, completely rescued this defect (Figure 2C, 2D). In addition, in the mouse developing limb where PCP plays an important role in limb elongation [24, 33, 38], induced wild type Vangl2 expression increased the length of the long bones in the Vangl $^{\text {low }}$ mutant. However, induced phospho-mutant Vangl2 expression further shortened the long bone (Figure 2E, 2F), possibly due to the dominant negative effects of phospho-mutant Vangl2 on the residual wild type Vang12 and Ror2 proteins. Taken together, these experiments indicate that Vangl2 phosphorylation is required for its function during mammalian development.

Vangl2 phosphorylation is required for its uniform asymmetric localization pattern and planar cell arrangement

A distinct character of established PCP is its uniformity throughout a tissue field of hundreds of cells, first reflected at a molecular level by the uniform asymmetric distribution of core PCP proteins. For example, Vangl2 is localized to the proximal side of chondrocytes in the developing limb [24]. This polarized localization pattern is difficult to recapitulate in cultured cells, most likely because of the lack of a stable Wnt gradient and full PCP machinery in vitro. To determine whether Vang12 phosphorylation is critical for its localization pattern and to reduce possible interference by endogenous wild type Vangl proteins, the Rosa-Vangl ${ }^{W t}$ and Rosa-Vang$12^{A}$ lines were crossed to the $\mathrm{Vangl1}^{-/-} ; \mathrm{Vangl2}^{-/-}$genetic background (designated as Vangl ${ }^{\text {null }}$ ). Detection of HA immunofluorescence in limb chondrocytes showed that the induced wild type Vangl2 protein recapitulated the uniform asymmetric localization pattern of endogenous Vangl2 along the limb's proximal-distal axis [24] (Figure 3A, 3B and Supplementary information, Figure S5A). However, the phospho-mutant Vang12 failed to show a polarized localization pattern and the orientation of asymmetric localization became randomized (Figure 3A, 3B). Similar to our observations in $\beta$-actin-Cre;Rosa-Van$g l 2^{A}$ mice (Figure 1G), phospho-mutant Vangl2 in the Vangl $l^{\text {null }}$ background also formed many discrete puncta (red arrows in Figure 3A), which may result from abnormal intracellular trafficking of phospho-mutant Vangl2 as suggested by transient expression assay in cultured cells (Supplementary information, Figures S2B and S3A). The phospho-mutant Vangl2 puncta are indeed co-localized with some vesicular markers including Lamp1 and Rab5 (Supplementary information, Figure S6). These puncta were rarely found in wild type HA-Vangl2 mice (Figure $3 \mathrm{~A})$. These results indicate that Vangl 2 phosphorylation is required for its uniform asymmetric localization pattern across the polarized tissues.

Given that the polarization of core PCP proteins di- 
A

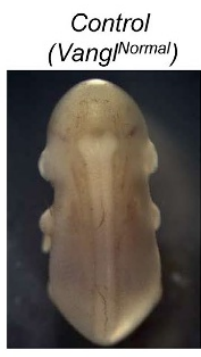

C

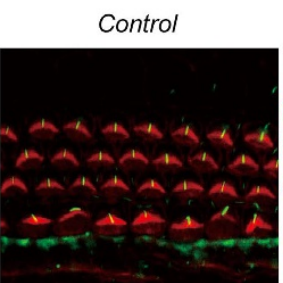

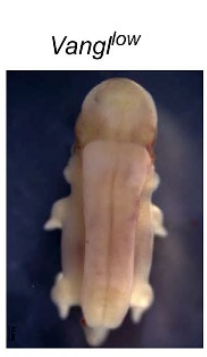

B-actin-Cre; Vanglow

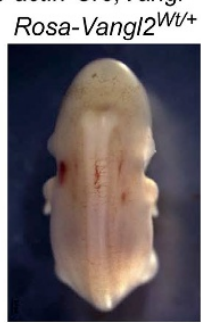

B-actin-Cre; Vanglow; Rosa-Vangl2 ${ }^{A /+}$

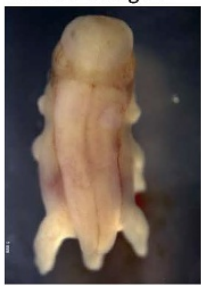

B-actin-Cre; Vanglow.

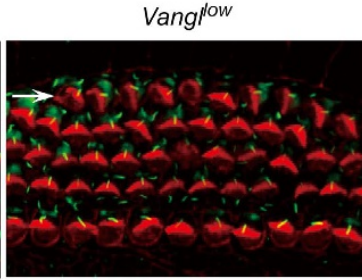

B-actin-Cre;Vanglow;Rosa-Vangl2 ${ }^{A /+}$
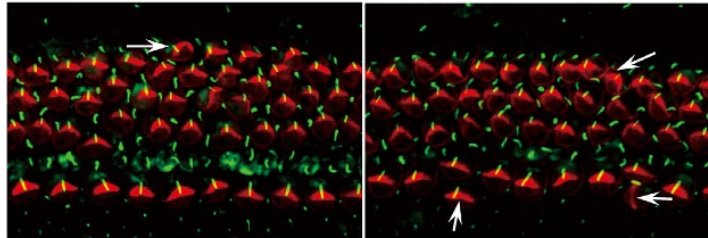
Rosa-Vangl2 ${ }^{W t}$

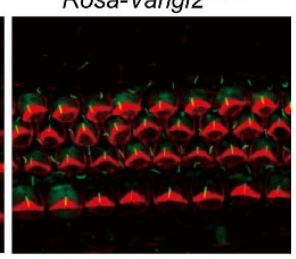

$E$

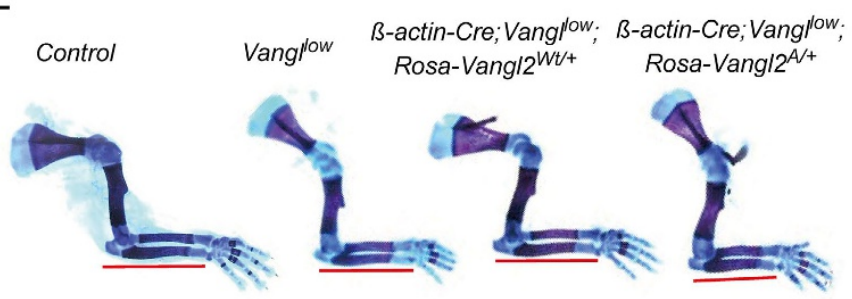

D

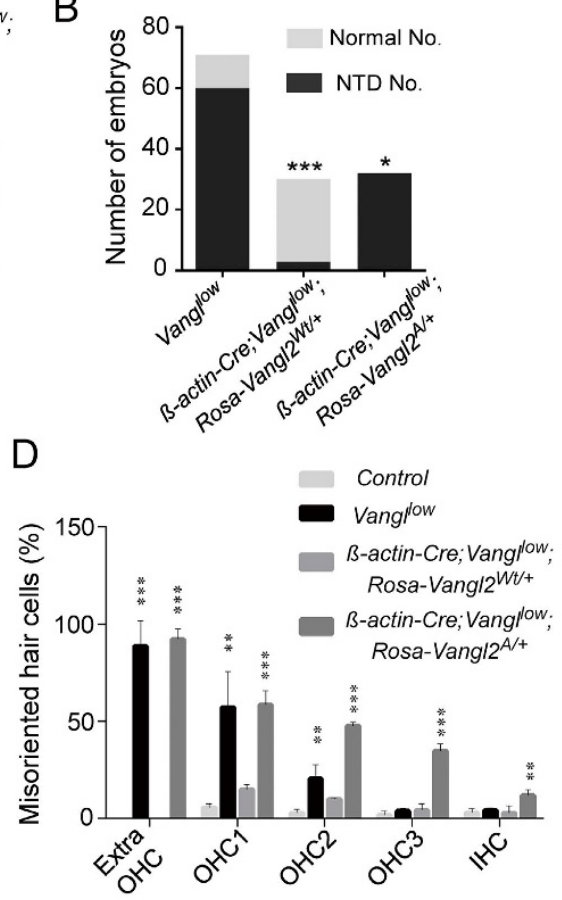

$\mathrm{F}$

B

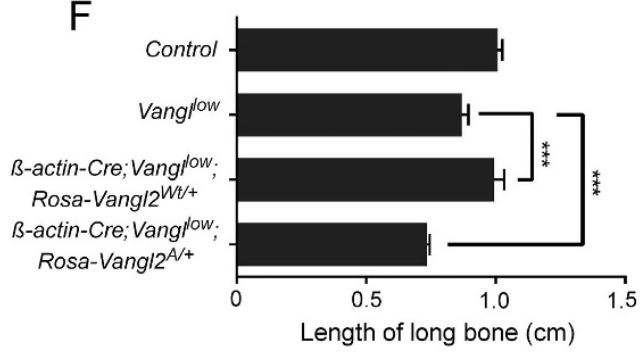

Figure 2 Vangl2 phosphorylation is required for PCP during mammalian development. (A) Neural tube defect of Van$\mathrm{g} / 1^{-/-} ;$Vangl2/2 $($Vanglow $)$embryos is rescued by $\beta$-actin-Cre-induced wild type Vangl2, but not phospho-mutant Vangl2. The

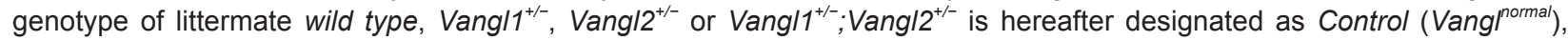
which are all morphologically normal and fertile. (B) The number of embryos analyzed in (A) in each genotype. NTD, neural tube defect. The complete list is shown in Supplementary information, Table S1. Fisher's exact test, ${ }^{* * *} P<0.0001$ or ${ }^{*} P<$ 0.05. (C) Immunofluorescent staining of actin-based stereocilium bundles (red, F-actin) and the microtubule-based cilia (green, acetylated tubulin) showing the organization of sensory hair cells in E18.5 inner ear cochlea. Arrows indicate additional hair cells. OHC, outer hair cells; IHC, inner hair cells. (D) Quantification of misoriented hair cells in embryos with different genotypes shown in (C). The cells with orientation angle $> \pm 30^{\circ}$ were considered as misoriented. $n=3$, error bars are means \pm SD. Total number of counted hair cells: Control $n=410$; Vangl ${ }^{\text {low }} n=670 ; \beta$-actin-Cre;Vanglow $;$ Rosa-Vangl $2^{\text {Wtt }} n=427 ; \beta$-actin-Cre;Vang ${ }^{\text {low }}$;Rosa-Vangl $2^{1 /+} n=573$. Two-tailed $t$-test, ${ }^{* * *} P<0.001$ or ${ }^{* *} P<0.01$. (E) Skeletal preparations of E18.5 embryonic forelimbs stained by alcian blue (cartilage) and alizarin red (bone). (F) The length of the ulna in (E) was measured. Control $n=4 ;$ Vanglow $^{\text {low }}=6 ; \beta$-actin-Cre;Vangl low $;$ Rosa-Vangl ${ }^{\text {Wtt }} n=5 ; \beta$-actin-Cre;Vanglow; Rosa-Vangl $2^{\beta /+} n=3$. Two-tailed $t$ test, ${ }^{* * *} P<0.001$. Error bars are means \pm SD.

rects polarized cellular behavior including coordinated control of cell shape and alignment, the shape and the orientation of chondrocytes in the distal limb were analyzed (Supplementary information, Figure S5A). In the control limb, distal chondrocytes are elongated, and the ratio of length to width (LWR, the length of the major axis of the cell divided by the length of the minor axis of the cell) is on the average of 1.9 (Figure 3C, 3D). These cells mostly orient their major axes perpendicular to the limb's P-D axis or $y$ axis $\left(-20^{\circ}\right.$ to $+20^{\circ}$ in reference to 
A

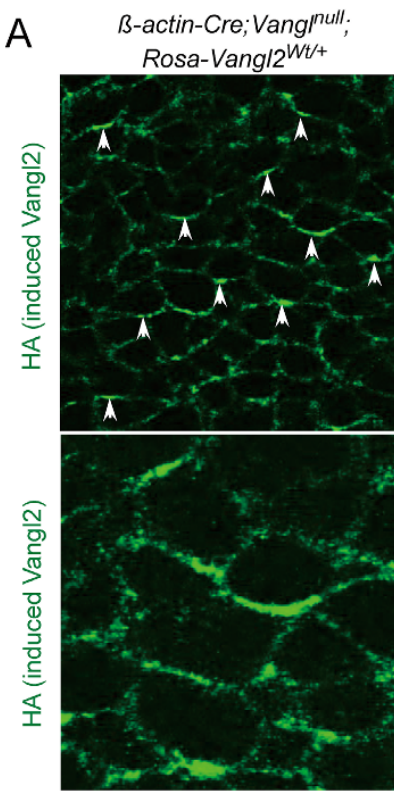

B

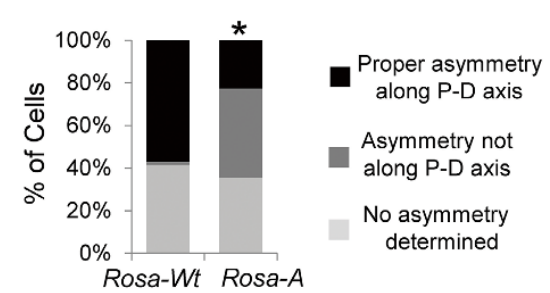

D

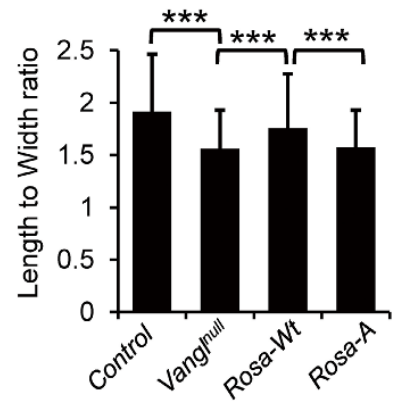

C

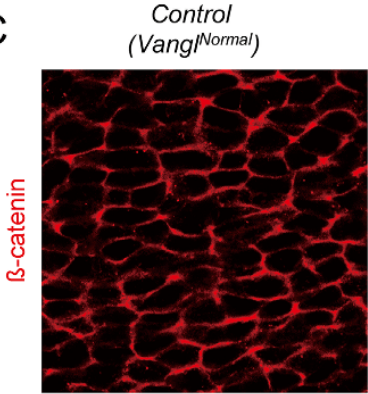

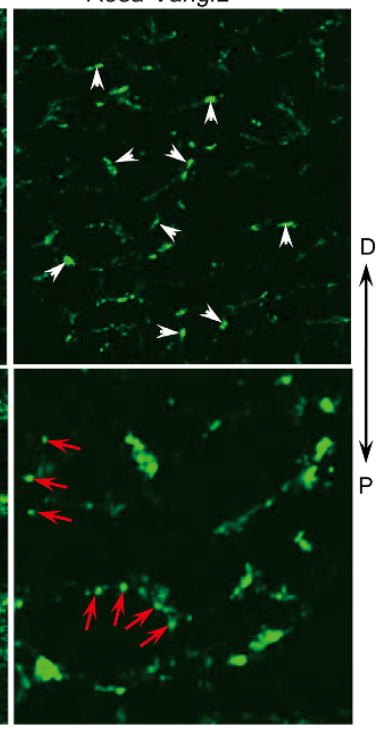

B-actin-Cre; Vangl ${ }^{\text {null }}$ Rosa-Vangl2 ${ }^{A /+}$
B-actin-Cre; Vangl ${ }^{\text {null; }}$; Rosa-Vangl2 ${ }^{W t /+}$

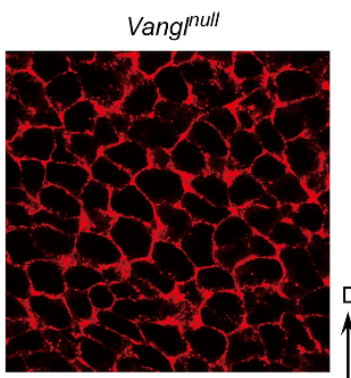

B-actin-Cre; Vangl Iull. $^{\text {. }}$ Rosa-Vangl2 ${ }^{A+}$
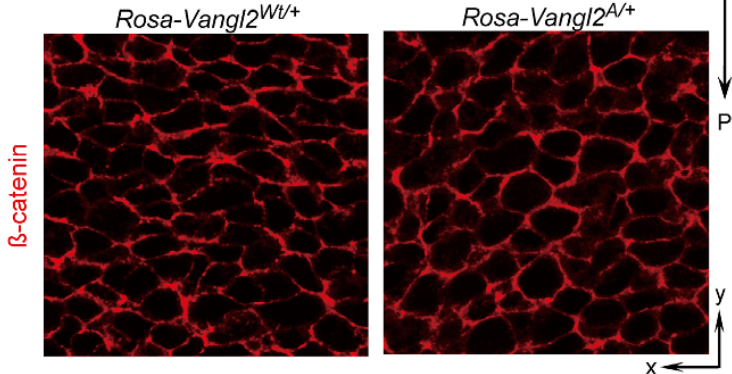

$E$

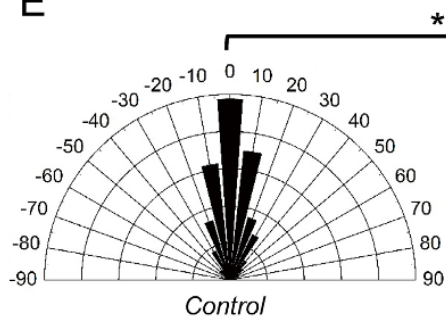

$* * *$

$0^{\circ}$
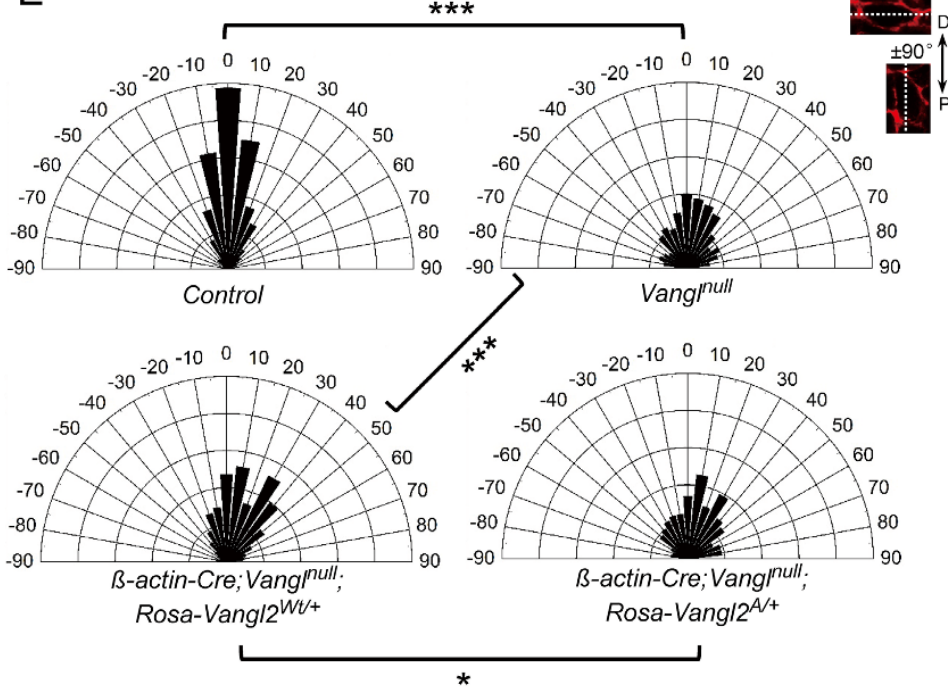

Figure 3 Vangl2 phosphorylation is required for its uniform asymmetric localization pattern and polarized cellular behaviors in limb chondrocytes. (A) HA staining of E13.5 distal limb chondrocytes showing unidirectional asymmetric localization of induced wild type HA-Vangl2 proteins (arrowheads in left panel) under endogenous Vangl1/2 null background (Vangl ${ }^{\text {null }}$ ). The localization of phospho-mutant Vangl2 proteins is randomized (arrowheads in right panel). The lower panels show magnified pictures and the red arrows point to many puncta formed by phospho-mutant Vangl2. P-D, proximal-distal axis of the limb. (B) The status of Vangl2 localization in (A) was counted and classified into three categories: proper asymmetric localization along the P-D axis (Vangl2 localized to the proximal side of the cell), asymmetric localization not along the P-D axis, and No asymmetry determined. The number of counted cells, $n=156$ and 132 for each genotype. Chi-square test, ${ }^{*} P<0.0001$. (C) Immunofluorescent staining of $\beta$-catenin (red) showing cell shape and orientation of E13.5 distal limb chondrocytes. The

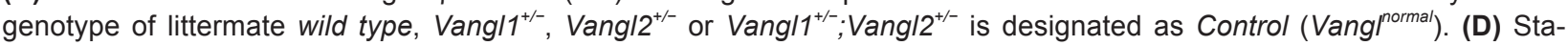
tistics of cell length to width ratio (LWR) of E13.5 distal limb chondrocytes. Significance among Control, Vang ${ }^{\text {rull }}$, Rosa-Wt

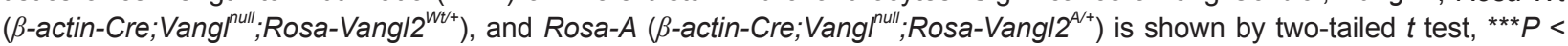
0.001 . Error bars are means $\pm S D$. (E) Schematic diagrams summarizing the quantification of E13.5 distal limb chondrocytes orientation. $x$ axis: angle of orientation; $y$ axis: percentage of cells at angle $x$. Horizontally oriented cells refer to $0^{\circ}$, vertically oriented cells along the P-D axis refer to $\pm 90^{\circ}$. Kolmogorov Smirnov test, ${ }^{* * *} P<0.001$ or ${ }^{*} P<0.05$. (D and E) $n>3$ for each genotype, the number of cells counted: Control, $n=730$; Vang ${ }^{\text {null }}, n=773$; $\beta$-actin-Cre; Vang ${ }^{\text {null }} ;$ Rosa-Vangl $^{\text {Wtt }}, n=364$; $\beta$-actin-Cre;Vang $\left.\right|^{\text {rull }} ;$ Rosa-Vangl $/ 2^{A /+}, n=474$. 
the $\mathrm{x}$ axis) (Figure 3E). However, in the Vangl $^{\text {null }}$ embryo, the cells were less elongated (the LWR is less than 1.5, close to 1.0 for round cells) and cell orientation became randomized (Figure 3C-3E). When wild type Vangl2 expression was induced in the Vangl ${ }^{\text {null }}$ background, the phenotype of cell shape and orientation was partially rescued. By contrast, phospho-mutant Vangl2 failed to do so and the mouse still showed less elongated and randomly oriented cells (Figure 3C-3E). Therefore, disruption of Vang12's global asymmetry by abrogating its phosphorylation also disrupted uniformly polarized cellular behavior.

Similarly, in skin epidermal cells that exhibit planar polarization [81], wild type HA-Vang12 was largely polarized along the anterior-posterior (A-P) body axis. However, the phospho-mutant HA-Vangl2 was randomly localized with multiple puncta (Supplementary information, Figures S5B and S7A). Accordingly, the shape and the orientation of skin epidermal cells were also changed significantly. The induced wild type Vang12, but not phospho-mutant Vang12, partially rescued the Vangl $l^{\text {null }}$ skin PCP phenotype (Supplementary information, Figure S7B-7D). Overall, these results indicate that Vang12 phosphorylation is required for its proper uniform asymmetric localization pattern and function during morphogenesis.

Function of Vangl2 phosphorylation in PCP is dose-dependent

Because Rosa-Vangl $2^{W t}$ and Rosa-Vangl2 ${ }^{A}$ were not expressed from the endogenous Vangl2 locus, we sought to generate a phospho-mutant Vang12 mouse model by mutating the endogenous Vangl2 gene. A Vangl2 ${ }^{3 A}$ allele was, therefore, generated by mutating the codons of the three Vang12 phosphorylation priming sites (S5, S82, and S84) to that of Ala (Figure 4A). In cultured mammalian cells, mutating all of these three priming sites (Vang12-3A) abolished Wnt5a-induced Vang12 phosphorylation [24]. Vang12-3A also exhibited similar dominant negative effects to phospho-mutant Vang12 (Vang12-A) (Supplementary information, Figure S3). Thus, we hypothesized that the Vangl $2^{3 A}$ line should behave similarly to the Rosa-Vangl $2^{A}$ line. However, to our surprise, Vangl $2^{3 A / 3 A}$ mutant mice were morphologically normal and fertile (Figure 4B). To test whether Vangl2 ${ }^{3 A}$ is a hypomorphic Vangl2 allele, we generated Vangl2 ${ }^{34 /-}$ mice. Interestingly, unlike the Vangl $2^{+/-}$mice that had no gross phenotypic defects, the Vangl $2^{34 /-}$ mice displayed head-shaking behavior (data not shown). This phenotype is similar to the previously described wobbly behavior of Vangl $2^{L p /+}$ mice $[82,83]$ and some of the other PCP mutants, such as Celsr1 mutants spin cycle and crash (Celsr1 is one of three mammalian orthologs of Fmi) [84]. We then further reduced the Vangl dosage by generating Van$\mathrm{gl1}^{+/-}$; Vangl2 $2^{3 A / 3 A}$ and Vangl1 $^{-/-}$; Vangl2 $2^{34 / 3 A}$ mice. While both Vangll $^{-/-}$and $\operatorname{Vangl}^{3 A / 3 A}$ mice were morphologically normal, three out of seven Vangl1 ${ }^{+/-}$; Vangl2 ${ }^{3 A / 3 A}$ mice showed a loop tail phenotype (all females showed vaginal agenesis), and Vangl1 $^{--}$; Vangl2 ${ }^{3 A / 3 A}$ mutant (three out of four) exhibited open neural tube defects (Figure 4B). In the inner ear cochlea, organization and orientation of sensory hair cells in the Vangl1 $1^{-/-}$;Vangl2 $2^{3 A / 3 A}$ mutant embryos was also disrupted (Figure $4 \mathrm{G}, 4 \mathrm{H}$ ). These results demonstrate that in mammalian development, Vangl2 ${ }^{3 A}$ is hypomorphic and phosphorylation is required to regulate Vangl2 activity.

The phenotype discrepancy between Rosa-Vangl $2^{A}$ and Vangl2 ${ }^{3 A / 3 A}$ lines and the Vangll dose-dependent effect of Vangl $2^{3 A / 3 A}$ mice suggested that Vangl2-3A has only partially lost its activities. Although Wnt5a failed to induce phosphorylation of Vangl2-3A in vitro [24], Vangl2-3A phosphorylation may not have been completely lost in vivo. We therefore examined Vang12's phosphorylation status in Vangl2 ${ }^{3 A / 3 A}$ mice (Figure 4C). Interestingly, although Vangl2 phosphorylation on S82 and S84 did not occur (indicated by phospho-specific antibodies), the total Vangl2 phosphorylation (indicated by band shift) was not completely abolished as we had originally expected, albeit partially reduced, in the $\operatorname{Vangl} 2^{3 A / 3 A}$ mutants. These data indicate that Vangl2 ${ }^{3 A}$ is a hypophospho-Vangl2 mutant.

The receptor tyrosine kinase Ror2 has been shown to mediate Wnt5a-induced Vang12 phosphorylation by binding to Wnt5a and forming a complex with Vang12 [24]. Ror2 and Vangl2 genetically interact with each other. Ror2 $2^{-/-}$; $\operatorname{Vangl}^{l p /+}$ (Lp, loop tail, S464N [82, 83]) embryos showed neural tube defects [24]. To test whether Vangl $2^{3 A}$ also enhances the phenotypes of the Ror $2^{-/-}$ mice, we generated $R o r 2^{-/} ; \operatorname{Vang} l 2^{3 A / 3 A}$ double mutant mouse embryos and found that the reduction of Vangl2 phosphorylation significantly enhanced the shortened tail and limb phenotypes of the Ror $2^{-/-}$mutant (Figure 4D-4F). The Ror2 ${ }^{-/} ;$Vangl2 $^{3 A / 3 A}$ double mutant showed almost no tail (Figure 4D). These mice also showed neural tube defects at low penetrance (data not shown). The long bones of $\operatorname{Ror}^{--} ; \operatorname{Vangl} 2^{3 A / 3 A}$ were further shortened compared with Ror $^{-/-}$(Figure 4E, 4F). In the inner ear cochlea, while the organization / orientation of sensory hair cells were largely normal in both $\operatorname{Vangl}^{34 / 3 A}$ and $\operatorname{Ror2}^{-/-}$embryos, it was impaired in $\operatorname{Ror2}^{-/-} ; \operatorname{Vangl} 2^{3 A / 3 A}$ double mutant (Figure 4G, 4H).

Taken together, these genetic analyses of $\operatorname{Vangl} 2^{3 A}$ mice revealed that $\operatorname{Vangl}^{3 A}$ is a hypomorphic Vangl2 allele that exhibits reduced phosphorylation, further confirming that Vang12 phosphorylation is required for 
A

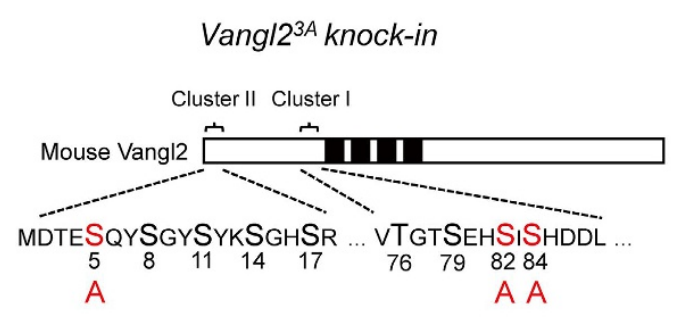

B

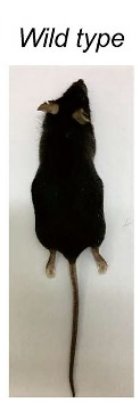

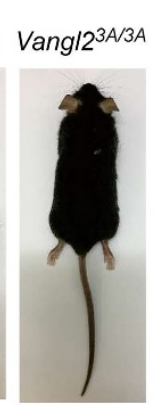
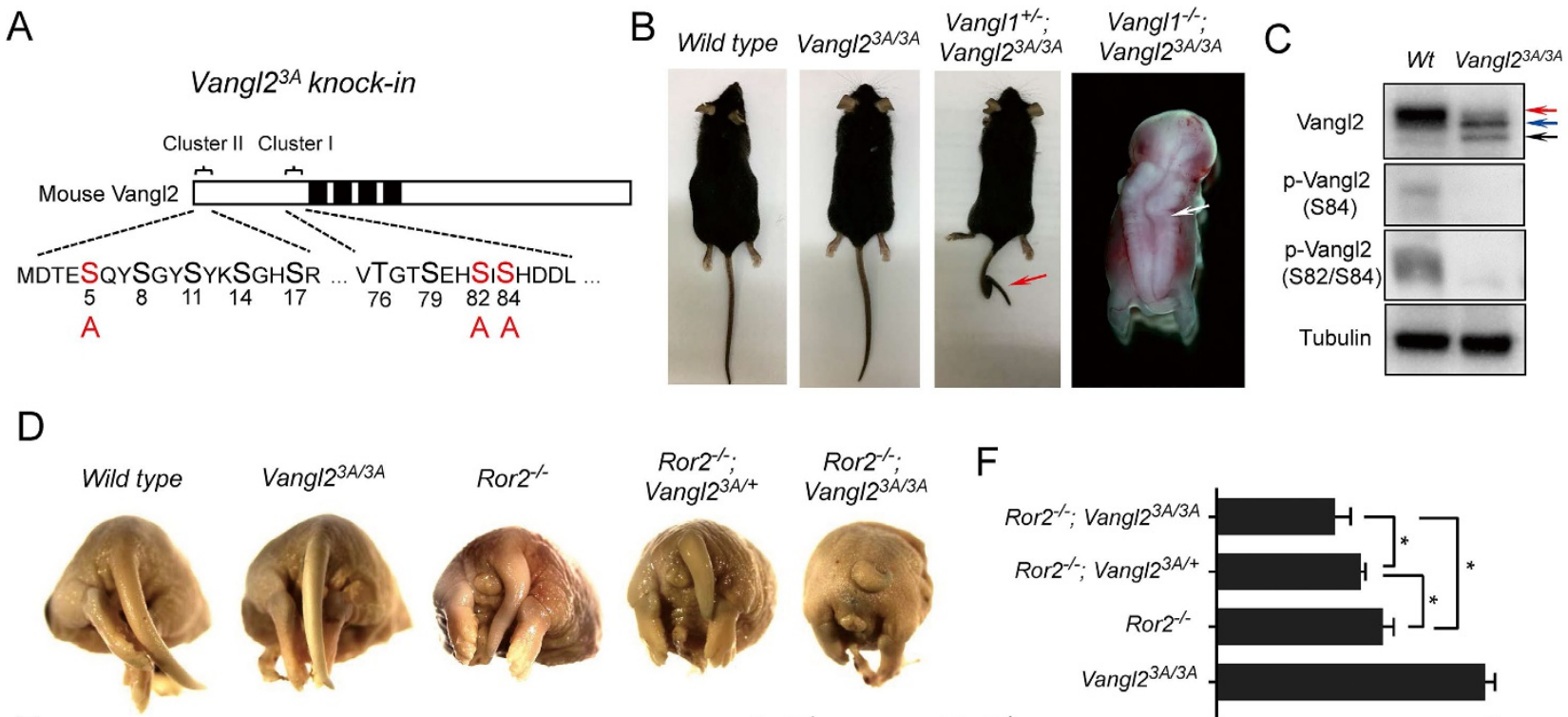

Ror $^{-/-}$;

Vangl $2^{3 A} / 3 A$

F
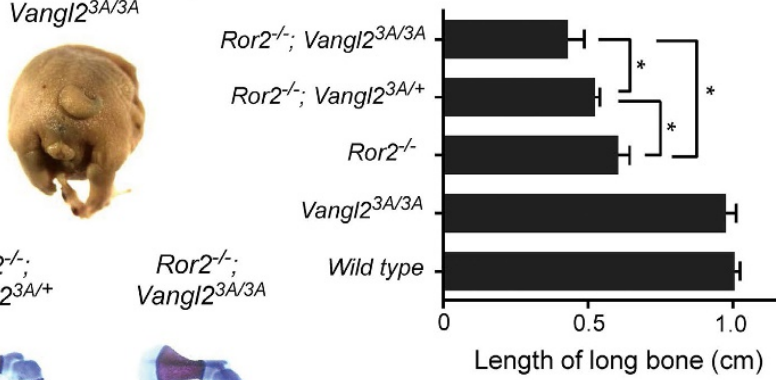

E

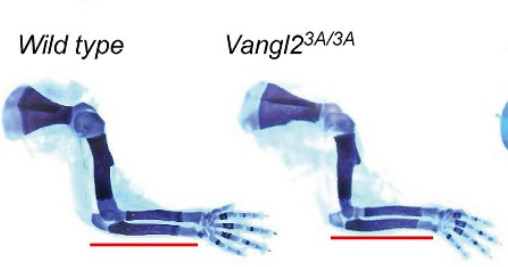

Ror2 ${ }^{-/-}$

Ror2 $^{-1-\text {; }}$ Vangl $2^{3 \mathrm{~A} /+}$
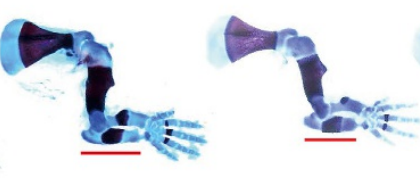

Length of long bone $(\mathrm{cm})$

G
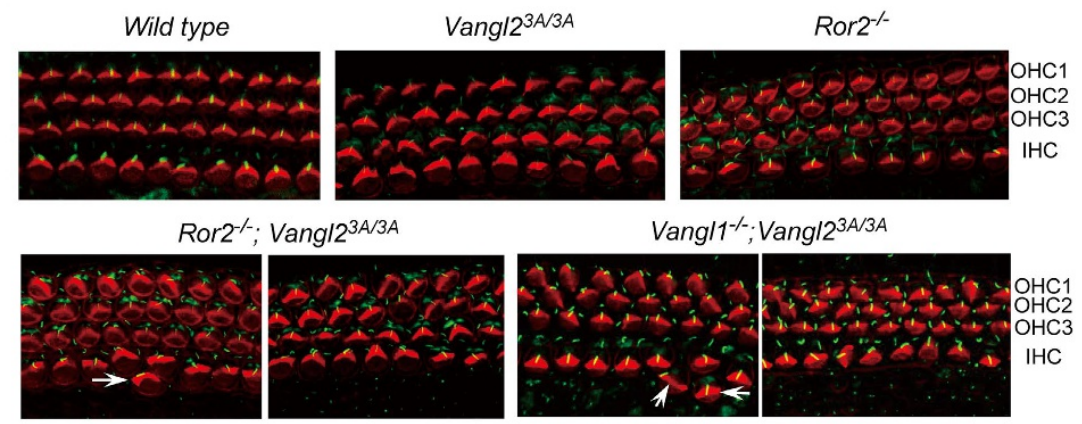

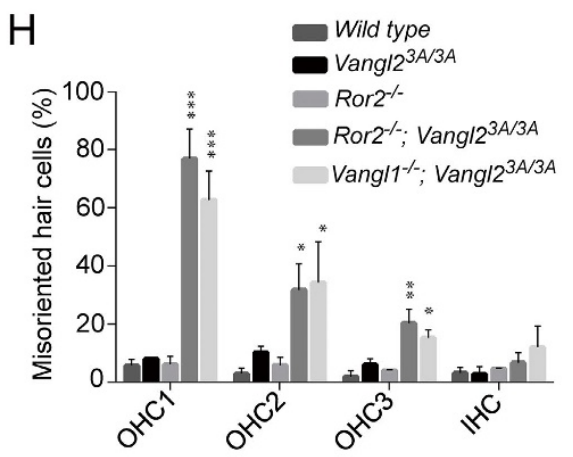

Figure 4 Hypophosphorylated Vangl2 shows reduced activities in PCP. (A) Generation of a phospho-mutant Vangl2 ${ }^{3 A}$ model by mutating the endogenous Vangl2 locus. The codons for three serines (S5, S82, S84) are altered to encode alanines (3A mutation). (B) Loss of one allele of Vangl1 in Vang/2 $2^{3 A / 3 A}$ led to loop tail (red arrow) in 3/7 mice; all females also had vaginal agenesis. Loss of two alleles of Vangl1 in Vang/2 ${ }^{3 A / 3 A}$ caused open neural tube defects (white arrow) in 3/4 embryos. (C) Western blot analysis of E11.5 Vang/2 ${ }^{3 A / 3 A}$ embryos. The phosphorylation shift of Vangl2 is reduced and the phosphorylation on S82 and S84 is abolished. The red, blue, and black arrows point to different levels of Vangl2 phosphorylation. (D) The E18.5 embryonic tails with indicated genotypes. It is nearly lost in Ror2 ${ }^{-/-}$; Vangl $2^{3 A / 3 A}$ mutant (5/5). (E) Skeletal preparations of E18.5 forelimbs stained with alcian blue (cartilage) and alizarin red (bone). The presence of Vangl $2^{3 A}$ alleles further shortened the length of the Ror2 ${ }^{-/-}$long bone. (F) The length of the ulna in (E) was measured. Wild type $n=4 ;$ Vangl $2^{3 A / 3 A} n=4 ; R o r 2^{-/-}$ $n=3$; Ror $2^{-/-} ;$Vang $/ 2^{3 A /+} n=4 ;$ Ror $2^{-/-} ;$Vang $/ 2^{3 A / 3 A} n=5$. Two-tailed $t$ test, ${ }^{*} P<0.05$. Error bars are means $\pm S D$. (G) Immunofluorescent staining of actin-based stereocilium bundles (red, F-actin) and the microtubule-based cilia (green, acetylated tubulin) showing the organization of sensory hair cells in E18.5 inner ear cochlea of indicated genotypes. The orientation and arrangement of sensory hair cells are disrupted in $R o r 2^{-/-} ;$Vangl $2^{3 A / 3 A}$ and $V a n g / 1^{-/-} ;$Vangl $2^{3 A / 3 A}$ embryos. Arrows point to extra hair cells. OHC, outer hair cells; IHC, inner hair cells. (H) Quantification of misoriented hair cells in embryos with different genotypes shown in (G). The cells with orientation angle $> \pm 30^{\circ}$ were considered as misoriented. $n \geq 3$, error bars are means \pm SD. Total number of counted hair cells: Wild type $n=410 ;$ Vangl $2^{3 A / 3 A} n=194 ;$ Ror $^{-/-} n=194 ;$ Ror2 $^{-/-} ;$Vangl $2^{3 A / 3 A} n=374$; Vang $/ 1^{-1-} ;$ Vangl $2^{3 A / 3 A} n=295$. Two-tailed $t$-test, ${ }^{* * *} P<0.001,{ }^{* *} P<0.01$ or ${ }^{*} P<0.05$. 
mammalian PCP and that phosphorylation levels control the activity levels of Vangl2 in PCP in vivo. The priming sites (S5, S82, and S84) previously identified in vitro are not strictly required for Vangl2 phosphorylation at other sites in vivo. This likely reflects the absence or reduced activities of kinases that can also phosphorylate Vangl2 without priming or due to an incomplete set of molecules involved in Wnt/PCP in cultured cells. Interestingly, this finding is different from the recent findings in Drosophila in which mutating two of the phosphorylation sites S120 and S122, corresponding to two of the priming sites in mouse Vang12, S82, and S84 (Supplementary information, Figure S1), was sufficient to abolish the Vang's phosphorylation and function in Drosophila PCP [70].

$C K 1 \varepsilon / \delta$ and Dvl are required for most of the phosphorylation events in Vangl2

In our previous studies, CK $1 \delta$ was identified as a potential kinase candidate that can induce Vang12 phosphorylation [24]. Interestingly, the phosphorylation site clusters in Vang12 are CK1 consensus sites (pS/T-X-X-S/ $\mathrm{T})$. This result was supported by recent studies in Drosophila, wherein CK1 $\varepsilon$ was found to phosphorylate Vang [70]. In mammalian cells, the CK1 inhibitor D4476 [85] strongly inhibited Wnt5a-induced Vangl2 phosphorylation (first row of Figure 5A). Notably, however, Vangl2 phosphorylation was not completely abolished following treatment with D4476 with or in the absence of Wnt5a induction (blue arrows in the first row of Figure 5A). To determine the sites of phosphorylation mediated by CK1, several mutant Vangl2 constructs were employed and tested (Figure 5A, see Supplementary information, Figure S1 for distribution of phosphorylation sites). First, we mutated either only the S84 priming site to Ala (S84A) or S84 together with all of cluster II sites to Ala (S5 17,84A). We found that Wnt5a-induced phosphorylation of S84A or S5 17,84A Vang12 was completely abolished by D4476 treatment (second and third rows of Figure $5 \mathrm{~A}$ ). These results suggest that phosphorylation of all cluster II and cluster I sites, except S84, requires CK1. To further test this, we generated S5 17,76 82A mutant Vang12, in which all phosphorylation sites except S84 were mutated to Ala. Phosphorylation of this mutant Vang12 was not inhibited by D4476 (blue arrow at the bottom row of Figure 5A). Interestingly, Wnt5a appeared to enhance the phosphorylation of the S5 17,76 82A mutant Vangl2 (indicated by the blue arrow) even with D4476 treatment. This suggests that Wnt5a promotes S84 phosphorylation of Vang12 in a CK1-independent manner. These data indicate that phosphorylation of most cluster I and II sites in Vangl2 depends mainly on CK1, whereas other kinase(s) phosphorylate S84. This is dis- tinct from CK1 regulation of Drosophila Vang, where CK1 $\varepsilon$ was suggested to phosphorylate both S120 and $\mathrm{S} 122$, sites that correspond to mouse S82 and S84 [70] (Supplementary information, Figure S1).

To identify the subfamily members of CK1 responsible for Wnt5a-induced Vangl2 phosphorylation, we referred to the studies in Drosophila. Discs overgrown (dco, Drosophila homolog of the $C K 1 \varepsilon / \delta$ subfamily) is required for PCP $[86,87]$ and CK1 $1 \varepsilon$ phosphorylates core PCP protein Dsh/Dvl on multiple sites [88]. However, mutating several Ser/Thr residues of fly Dsh failed to reveal the critical CKI-regulated phosphorylation site(s) required for PCP [89]. These results suggest that other substrates may also mediate CK1's function in regulating PCP. A recent study shows that $d c o$ regulates PCP in Drosophila by phosphorylating Vang [70]. This finding is in agreement with results from our parallel study (Figure 5B), in which we tested the role of $\mathrm{CK} 1 \varepsilon$ and $\mathrm{Ck} 1 \delta$ in Vang12 phosphorylation in mammalian cells. This revealed that $\mathrm{CK} 1 \varepsilon$ and $\mathrm{CK} 1 \delta$ were redundantly required for $\mathrm{Wn}$ t5a-induced Vang12 phosphorylation. Hyperphosphorylated Vangl2 was diminished when $C K 1 \varepsilon / \delta$ kinases were knocked down (red arrow in Figure 5B). Interestingly, the Ser 84 phosphorylation detected by phospho-specific antibody appeared to be increased when both CK1 $\varepsilon$ and CK1 $\delta$ were knocked down (Figure 5B). This is likely due to the nature of phospho-specific antibody we generated. The Vangl2 phospho-S84 antibody was generated against the aa72-aa94 peptide containing only phosphorylated S84. Therefore, this antibody binds highly phosphorylated Vangl2 with low affinities when S84 neighboring sites (e.g., S79, S82) are also phosphorylated. Following $\mathrm{CK} 1 \varepsilon / \delta$ siRNA treatment, which reduced phosphorylation on all sites other than S84, the Vang12 phospho-S84 antibody would bind better to Vangl2 (lanes 2 and 6, Figure $5 \mathrm{~B}$ ). These results further support our conclusion that CK1 is obliged to phosphorylate other sites rather than S84. This led us to test the role of CK1 in Vangl2 phosphorylation further in $C K 1 \varepsilon$ and $C K 1 \delta$ mutant MEFs. In the absence of either $C K 1 \varepsilon$ or $C K 1 \delta$, recombinant Wnt5a still efficiently induced Vang12 phosphorylation (red arrows in Figure 5C). However, ablation of both CK1 $1 \varepsilon$ and $\mathrm{CK} 1 \delta$ almost completely abolished Wnt5a-induced Vangl2 phosphorylation (Figure 5C). Consistent with the data shown in Figure 5A, Vangl2 phosphorylation was not completely abolished either by $\mathrm{CK} 1 \varepsilon / \delta$ knockdown or in $C K 1 \varepsilon / \delta$ mutant MEFs (blue arrows in Figure 5B, $5 \mathrm{C})$. This is likely because of CK1-independent phosphorylation on S84 and/or the presence of other kinases that can also phosphorylate Vangl2.

To understand the functional significance of CK1 in regulating Vang12 phosphorylation, we first examined the 


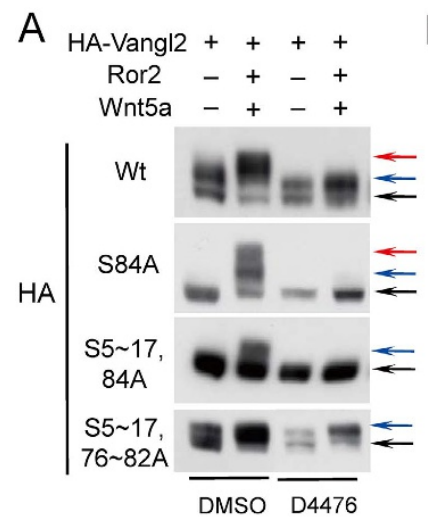

B

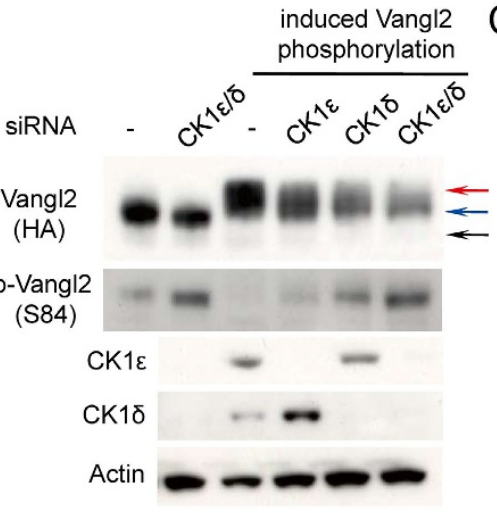

D

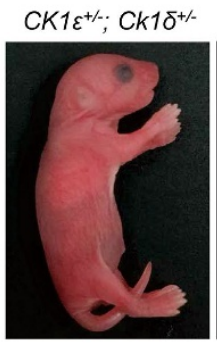

Vang/2Lp/+
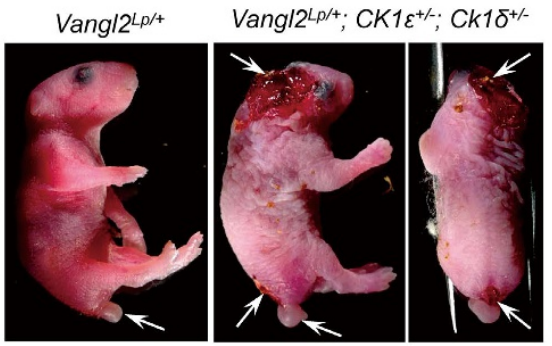

$E$

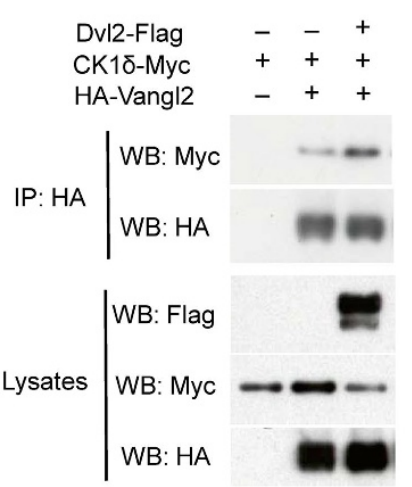

F

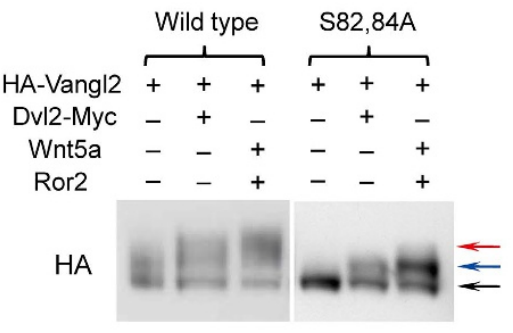

C

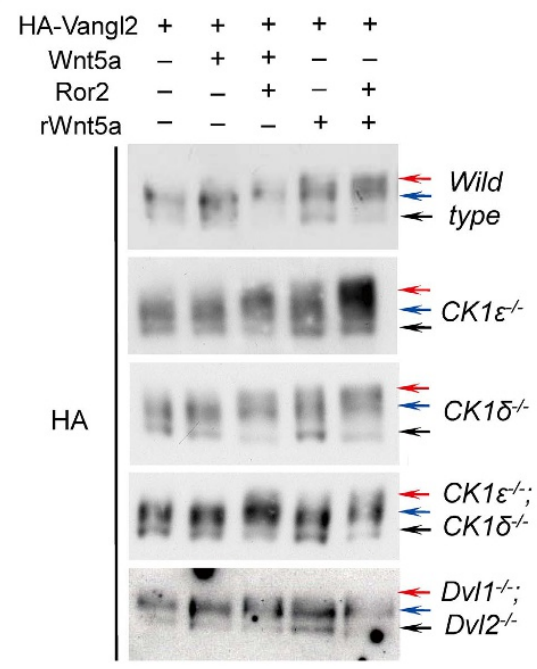

G

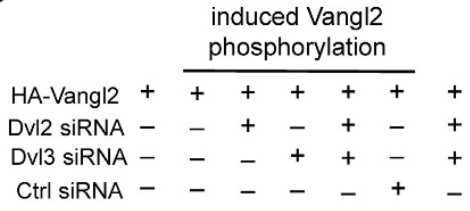

HA

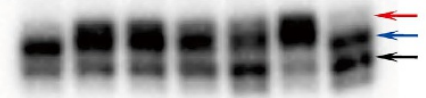

Dv12

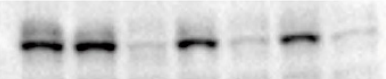

Dvl3

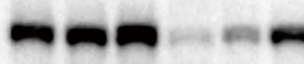

Tubulin

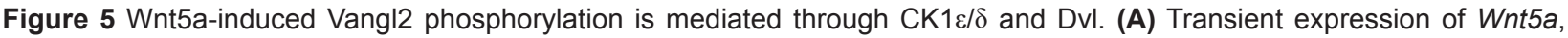
its receptor Ror2 and different forms of Vangl2 in CHO cells with or without CK1 inhibitor D4476 (100 $\mu$ M) treatment. D4476 inhibited Wnt5a-induced Vangl2 phosphorylation. (B) siRNA knockdown of CK1 $\varepsilon$ and/or CK1 $\delta$ in HEK293T cells. CK1 $\varepsilon$ and CK1 $\delta$ are redundantly required for induced Vangl2 phosphorylation. p-Vangl2 (S84) antibody specifically recognized the Vangl2 phosphorylation on S84, but the additional phosphorylation on neighboring sites (e.g. S79, S82) decreased its binding affinities. (C) Either Wnt5a/Ror2 plasmids were transfected or recombinant Wnt5a proteins (rWnt5a, 500 ng/mL, 2-h treatment) were added into wild type, $\mathrm{CK} 1 \varepsilon^{-/-}, \mathrm{CK} 1 \delta^{-/-}, \mathrm{CK} 1 \varepsilon / \delta$ double null or $D \mathrm{vl} 1 / 2$ double null MEFs to induce Vangl2 phosphorylation. (A-C) The red, blue, and black arrows indicate hyper-phosphorylated, hypophosphorylated, and unphosphorylated Vangl2, respectively. (D) Genetic interaction of Vangl2 and $C K 1$. The $C K 1 \varepsilon^{+/-} ; C K 1 \delta^{+/-} ;$Vangl2 ${ }^{L / /}$ mutant exhibited anterior open neural tube and spina bifida (arrows), $n=3$. (E) Co-immunoprecipitation between CK1 $\delta$ and Vangl2 in HEK293T cells. Expression of $D v / 2$ enhanced the CK1 $1 \delta$-Vangl2 binding. (F) Different forms of Vangl2 were co-transfected with Wnt5a, Ror2, or Dv/2 into CHO cells. Dvl2 alone induced Vangl2 phosphorylation. (G) siRNA knockdown of Dvl2 and/or Dvl3 in HEK293T cells. Dv/2 and $D v / 3$ are redundantly required for induced Vangl2 phosphorylation.

cellular localization of Vang12 in cultured cells treated with CK1 inhibitor D4476. Inhibition of CK1 significantly changed the pattern of Vang12 subcellular distribution. D4476 treatment reduced Vang12 localization on the cell membrane with or without Wnt5a induction
(Supplementary information, Figure S8A, S8B). We then further tested the genetic interaction between Vangl2 and $C K 1$ using the $C K 1 \varepsilon / \delta$ mutant mice and Vangl2 loop tail $(L p)$ mutant mice. The $C K 1 \varepsilon^{+/-} ; C K 1 \delta^{+/-}$mice were normal and Vangl2 $^{L p /+}$ mutant developed loop tail $(100 \%$ 
penetrance) and occasional spina bifida (5\%), whereas all the compound mutants $C K 1 \varepsilon^{+/-} ; C K 1 \delta^{+/-} ; \operatorname{Vang} l 2^{L p /+}$ $(n=3)$ exhibited both anterior and posterior neural tube closure phenotypes (arrows in Figure 5D). The genetic interaction of CK1 and Vangl2 in mammals is supported by similar findings in Drosophila where Vang and dco interact genetically in vivo [70]. CK1 is also a critical regulator of many other signaling pathways (e.g., Wnt/ $ß$-catenin, hedgehog signaling $[90,91])$, and CK1 family members exhibit functional redundancy [91, 92]. Thus, assessing the PCP phenotype genetically by further reducing CK1 doses in vivo in the mouse would be difficult. We have found that $C K 1 \varepsilon / \delta$ double mutant embryos died before E9.5 (data not shown) although $C K 1 \varepsilon$ or $C K 1 \delta$ single mutant mice showed abnormal circadian behavior with no morphological defects [92]. We, therefore, turned to an ex vivo limb culturing system, in which CK1 can be inhibited by its specific inhibitor D4476 only in the developing limb bud. In the wild type limb bud culture $(n=3)$, a high dose of D4476 $(20 \mu \mathrm{M})$ inhibited cartilage outgrowth and PCP (shown by polarized Vangl2 localization). Meanwhile, a low dose $(1 \mu \mathrm{M})$ showed no remarkable effect ( $n=3$, Supplementary information, Figure S9A-S9E). However, in the cultured Vangl2 ${ }^{3 A / 3 A}$ limb buds $(n=3)$, even the low dose of D4476 strongly inhibited cartilage growth and PCP $(n=3$, Supplementary information, Figure S9A-S9E). These results indicate that the limb phenotype of the hypomorphic Vangl $2^{3 A}$ mutant mouse embryo is strongly enhanced by inhibition of CK1 activities. These genetic results support our previous [24] and current biochemical studies that CK1 regulation of Vangl2 phosphorylation is essential for its role in PCP.

$\mathrm{Dsh} / \mathrm{Dvl}$ is an evolutionarily conserved core PCP regulator and it acts as a key scaffold protein [93]. Dvll;D$v l 2$ and $D v l 2 ; D v l 3$ double mutant mouse embryos display typical PCP defects including open neural tube and misoriented inner-ear hair cells [20, 22]. Given that Dvl genetically and physically interacts with Vangl2 [21, 22, 76], and that CK1 is known to bind and phosphorylate Dvl [88], we hypothesized that Dvl may also function to bring CK1 to phosphorylate Vang12. To test this hypothesis, we performed a co-immunoprecipitation experiment and found that CK1 bound to Vangl2. Such binding was significantly enhanced by Dvl (Figure 5E). Interestingly, overexpression of Dvl alone could induce Vang12 phosphorylation without Wnt induction. This effect is more obvious on S82,S84A mutant Vang12 (Figure 5F). It is interesting to note that these findings differ from those in Drosophila, where Dsh was unable to promote Vang phosphorylation, and $\mathrm{Fz}$ also failed to promote Vang phosphorylation when S120 and S122 (equivalent to mouse S82 and S84) were mutated [70]. Furthermore, by employing $D v l 1^{-/-} ; D v l 2^{-/}$mutant MEFs or knocking down $D v l 2$ and $D v l 3$, we confirmed that Dvl family members are required for Wnt5a-induced Vangl2 phosphorylation (Figure 5C and 5G). The level of unphosphorylated Vangl2 was increased when $D v l 2$ and $D v l 3$ were knocked down (lanes 5 and 7, pointed by the black arrow in Figure 5G). Taking all these results together, we have identified that $\mathrm{CK} 1 \varepsilon$ and $\mathrm{CK} 1 \delta$ are redundantly required for Wnt5a-induced Vang12 phosphorylation in PCP signaling. In contrast to Drosophila, Dvl is an important regulator of Vang12 phosphorylation in mammals.

\section{Discussion}

PCP provides directional information to control and coordinate polarized cellular behavior, which is fundamental for tissue morphogenesis and organ formation. However, it is unclear how the uniformity of the asymmetric localization of core PCP proteins, the most critical molecular event of PCP, is achieved. Two classes of modules, a global module and a local module, have been proposed [94]. The local module that describes the feedback amplification steps to establish local polarity in individual cells has been well studied in Drosophila [95, 96]. However, the global module that couples individual cellular polarity to the tissue axis is not well understood $[4,61]$. We and others provided genetic and biochemical evidence that Wnt gradients act as global cues to orient planar polarity in mouse [24, 68], Xenopus [66] and Drosophila [65]. Nevertheless, how the signal from a global cue is transduced to the core PCP proteins to break their symmetry still needs to be elucidated. In the past, the Fz activity gradient model has been well documented [49, 55]. In this model, a $\mathrm{Wg} / \mathrm{Wnt}$ gradient generates different levels of Fz activity in a dose-dependent manner. Then, each cell monitors the level of Fz activity in relation to its adjacent cells and orients itself toward its neighbor with low Fz activity. However, it is unclear how Fz activity is defined in this model. Fz activity may be modulated by Fz internalization upon binding to Wnt ligands [97] or through interaction with Vang, another essential component of PCP, across the plasma membrane of neighboring cells [98]. Vang is eventually polarized to the opposite side of $\mathrm{Fz}$ within the cell. Our previous studies found that Vangll and Vangl2 genes are dose-dependently required for mammalian PCP [19] and that Wnt5a can directly regulate the level of Vang12 phosphorylation through Ror2 in a Wnt5a dose-dependent manner [24]. In this study, we provide genetic evidence that Wnt-induced Vangl2 phosphorylation is required for its uniform polarization and its function in PCP during mammalian 
development. In addition, we show that phosphorylation levels are important for defining Vang12 activities in vivo. Hypophosphorylated Vangl2 displays reduced activities and a phospho-mutant Vangl2 exhibits dominant negative effects on its binding partners. These findings provide a molecular mechanism, whereby responding cells interpret distinct Wnt5a dosages through the Vangl2 phosphorylation level. Thus, our work provides the first evidence that Wnt regulates Vangl through phosphorylation in vivo to provide a critical signaling event in establishing mammalian PCP. This supports a Vangl activity gradient model in transducing Wnt/PCP signaling.

During the preparation of this manuscript, Kelly et al. [70] reported that Vang phosphorylation is also required for its polarized distribution and PCP in Drosophila development. However, the regulation and functional requirement of Vang or Vangl2 phosphorylation on priming sites differ in Drosophila and mammals. First, although Drosophila Vang also contains the conserved Ser and Thr sites organized in two clusters similar to those of mammalian Vangl2 (Supplementary information, Figure S1), only S120 and S122 (corresponding to S82 and S84 in mouse) were identified as required for Vang phosphorylation and PCP in Drosophila [70]. By contrast, disruption of mouse Vang12 phosphorylation on S5, S82, and S84 (equivalent to Drosophila S5, S120, and S122) is hypomorphic with no obvious PCP defects. Phosphorylation on some other sites in the two clusters still occurs and the partially phosphorylated Vangl2 still displays activity. Second, Drosophila CK1 $1 \varepsilon$ was suggested to phosphorylate both S120 and S122 [70]. However, in mammalian cells, kinase(s) other than $C K 1 \varepsilon / \delta$ regulate Vang12 phosphorylation on S84 (equivalent to Drosophila S122). This difference suggests additional complexity to PCP regulation at the level of Vangl2 phosphorylation in mammals. An unanswered question in Drosophila PCP is whether $\mathrm{Wg} / \mathrm{Wnt}$ ligands can induce Vang phosphorylation, even though Fz can promote Vang phosphorylation in a cell-autonomous manner [70]. Third, compared with the essential role of S120/S122 phosphorylation in Drosophila PCP, the phosphorylation induced by Wnt5a on sites other than S82/S84 in mammals is partially independent of S82/S84 and also functionally important. Further loss of phosphorylation at these sites in addition to S82/S84 resulted in much more severe PCP defects. These observations in mice provide mechanistic insights into human defects that are associated with human $V A N$ $G L$ mutations. Mutation in human VANGL1 on S83 (equivalent to mouse Vang12 S79 and Drosophila S117) is associated with a neural tube defect [71]. Together, the genetic studies in Drosophila and mouse demonstrate that Vang/Vangl phosphorylation is an evolutionarily conserved critical event in PCP. However, the regulatory and functional mechanisms have become more complex with the evolution of Wnt/PCP signaling from Drosophila to mammals. In contrast to the limited Vang phosphorylation events and unknown functions of $\mathrm{Wg} /$ Wnt ligands in regulating Vang phosphorylation in Drosophila, mammals might have evolved a Wnt5a dependent system, in which the dose of Wnt5a quantitatively determines Vangl2 activities by inducing different levels of Vangl2 phosphorylation in two clusters of Ser and Thr sites. This evolutionary difference in Wnt/PCP signaling might reflect the requirement for a Wnt signaling gradient to induce PCP in tissues of a larger size in mammals. One can imagine that in a tissue field with a large landscape, Vangl2 activities with fine interval changes are needed to convey information provided by different Wnt doses in the gradient in order to orient the cells in one direction.

In addition to Vang/Vangl phosphorylation, new regulatory schemes have been developed to regulate mammalian PCP. For example, besides the canonical Frizzled family of Wnt receptors, other Wnt receptors, such as Ror2, Ryk, and Ptk7, are also required for PCP in the mouse [23-26, 99]. Ror2 enhances Wnt5a-induced Vang12 phosphorylation [24] and Ryk regulates Vangl2 by regulating its protein stability [26]. While Drosophila Fz can strongly promote Vang phosphorylation, it is surprising that the $\mathrm{Fz} / \mathrm{PCP}$ signaling mediator Dsh cannot regulate Vang phosphorylation [70]. By contrast, we found that mammalian Dvl promotes Vangl2 phosphorylation by enhancing the interaction between CK1 and Vang12. Since Wnt5a/Ror2 strongly promotes Vangl2 phosphorylation [24] and recruits Dvl to the cell membrane [100], it appears that the mammals have evolved a new mechanism for regulating Vangl2 phosphorylation through Dvl. Interestingly, human WNT5A and ROR2 mutations, identified as causing Robinow syndrome [4244], exhibited reduced activity in promoting Vang12 phosphorylation [24]. DVL1 and DVL3 mutations also cause Robinow syndrome [45, 46], supporting the notion that Dvl, similar to Ror2, also promotes Wnt5a-induced Vangl2 phosphorylation in mammals. Ror2 and Vang12 or Ror2 and Fzd were, respectively, found to form a receptor complex to mediate noncanonical Wnt signaling $[24,101]$ and both Fzd and Ror2 can bind Dvl [100, 102]; thus, one possible scenario in the mammalian system is that Wnt ligands bind to both Fzd and Ror2, which recruit CK1 through Dvl to phosphorylate Vang12. CK1 may contribute to Wnt/PCP signaling through phosphorylating both Dvl and Vangl2.

Vesicle trafficking of core PCP proteins has been implicated in regulating PCP [103-109]. Our current study 
suggests that phosphorylation-regulated endocytosis of Vang12 may also play a critical role in establishing PCP. Transiently overexpressed phospho-mutant Vang12 strongly colocalizes with endocytic vesicles in cultured cells. Low levels of phospho-mutant Vangl2 expressed from Rosa 6 locus also formed multiple discrete puncta in vivo and exhibited dominant negative effects on its binding partners. The random localization pattern of phospho-mutant Vang12 in tissues in vivo might result from its dysregulated intracellular trafficking and/or its abnormal interaction with other PCP regulators. Given the graded expression pattern of Wnt5a during mammalian development [62-64], different concentrations of Wnt5a molecules in neighboring cells may induce different levels of Vangl2 phosphorylation, resulting in different amounts of Vangl2 proteins stabilized at the cell surfaces of the neighboring cells through intracellular trafficking. Such an initially small difference between neighboring cells may break the original symmetric distribution of Vangl2 by known antagonism between Fz/Dsh/Dgo and Vang/Pk protein complexes, leading to their eventual polarized localization pattern $[8,27,95,96]$. Studies on Vang/Vangl phosphorylation have begun to reveal how PCP is initiated and established by global polarizing cues. Possibly, Wg/Wnt gradients simultaneously generate both Fz/Fzd activity and Vang/Vangl activity gradients to break the symmetry of core PCP protein distribution. However, some fundamental questions remain. Further studies are warranted, for example, to understand the precise mechanisms of how Wnt-regulated Vang12 phosphorylation leads to its polarized localization and how Vangl2 and Fzd proteins finally segregate to the opposite sides of the cell, while Ror2 does not display any asymmetric localization.

\section{Materials and Methods}

\section{Mouse lines, breeding and genotyping}

The Vangl1 ${ }^{+-}$, Vangl2 ${ }^{+-}$, and Ror $2^{+/-}$mouse strains and methods for their genotyping have been described previously [19, 24]. The inducible Vangl2 lines (Rosa-Vangl2 ${ }^{W t}$ and Rosa-Vangl2 ${ }^{4}$ ) were generated by knock-in of the wild type or phospho-mutant Vangl2 cDNA into the Rosa26 locus (Figure 1C). The 3× HA-tagged Van$g l 2$ cDNA was first inserted downstream of the PGKneotpAlox2 cassette in a plasmid obtained from Addgene (Addgene \#13444). The whole fragment containing the loxp-flanked neo cassette and HA-Vangl2 was then released and inserted into the pROSA26-1 plasmid (Addgene \#21714) to complete the Rosa-Vangl2 targeting constructs. The Vangl2 phospho-compromised mouse line (Van$g l 2^{34}$ ) was generated by direct knock-in to change serines at S5, 82 , and 84 into alanines. The targeting construct previously used to generate the Vangl2 conditional line [19] was used as mutagenesis template. Gene targeting was performed in 129Sv/C57B6 hybrid embryonic stem (ES) cells. G418-resistant clones with the appropriate homologous recombination events were confirmed by long range PCR. Mouse chimeras were generated from targeted ES cells by morula aggregation and were further crossed with wild type C57B6 mice to generate F1: Rosa-Vangl2 ${ }^{\text {wt/ }}$, Rosa-Vangl2 ${ }^{A /+}$, or Vangl2 ${ }^{3 A /+}$. F1 mice were intercrossed to generate homozygous mice or crossed to other mouse lines to generate compound mutants. To induce Rosa-Vangl2 expression in the Vangl1 and Vangl2 mutant background, Sox2-Cre or $\beta$-actin-Cre; Vangl1 ${ }^{+/-}$:Vangl2 ${ }^{+-}$ mice were first generated and then crossed to Rosa-Vangl2 $2^{W t / W t}$; Vangl1 $^{-/-} ; V_{\text {Vangl2 }}{ }^{c / c}$ or Rosa-Vangl2 $2^{A / A} ;$ Vangl $^{-/-} ;$Vangl2 $^{c / c}$ mice. The genotype of littermate wild type, Vangl1 ${ }^{+-}$, Vangl2 $2^{+-}$or Vangl1 $^{+-} ;$Vangl2 $^{+/-}$is designated as Control (Vangl ${ }^{\text {mormal }}$ ) because they are all morphologically normal, healthy, and fertile. As we maintained all mouse lines by crossing with C57B6 background mice (for more than eight generations), the genetic background of embryos used in this study is considered as C57B6. The primers used to genotype Rosa-Vangl2 lines: Rosa-Vangl2-P1, CCAAAGTCGCTCTGAGTTGTTAT; Rosa-Vang12-P2, ACACACCAGGTTAGCCTTTAAGC; and Rosa-Vangl2-P3, CAAGTCACATAAGTTCGTCATGC. The wild type construct is $255 \mathrm{bp}$; the targeted construct, $500 \mathrm{bp}$. The primers used to genotype Van$g l 2^{3 A}$ line: Vangl2-3A-F: CCATCGATGCACATGTGGTATC and Vangl2-3A-R: GACACTGTCCTCCATGTCCTTG. The wild type construct is $330 \mathrm{bp}$; the targeted construct, $400 \mathrm{bp}$. The Vangl2 $2^{L p /+}$, $C K 1 \varepsilon^{c / c}$, and $C K 1 \delta^{c / c}$ conditional mouse strains were purchased from Jackson Laboratory. $C K 1 \varepsilon^{-/-}, C K 1 \delta^{-/-}$, and $C K 1 \varepsilon^{-1-} ; C K 1 \delta^{-/-}$ MEFs were generated by infection with adenovirus expressing Cre recombinase. $D v l 1^{-/-} ; D v l 2^{-/-}$MEFs were isolated from the $D v l 1 / 2$ double mutant mouse embryos.

\section{Plasmids and cell culture}

The Vangl2, Vangl1, Ror2, and Wnt5a expression plasmids have been described previously [24]. The Myc tag or Flag tag was fused to Dv12 protein. The CK1 $\delta$ expression plasmid was provided by Jeffrey Rubin. Dynamin and RFP-tagged Clathrin, Caveolin, Rab5, Rab7, and Lamp1 expression plasmids were obtained from Addgene. CHO, HEK293T, MDCK, and MEF cells were incubated in DMEM containing 10\% FBS, penicillin G (100 units $/ \mathrm{mL})$, and streptomycin $(100 \mu \mathrm{g} / \mathrm{mL})$ at $37{ }^{\circ} \mathrm{C}$ in a humidified $5 \% \mathrm{CO}_{2}$ incubator. For co-transfection experiment, equivalent amounts of plasmids (300 ng per plasmid in 24-well plate) were transfected using Lipofectamine 3000 reagent following the manufacturer's recommendation. Transfected cells were then cultured for $48 \mathrm{~h}$ and harvested for immunoblot or immunofluorescent staining.

\section{Immunocytochemistry}

Flag, HA, or YFP-tagged Vangl2, Vangl1, Ror2, or Dynamin and RFP-tagged Clathrin, Caveolin, Rab5, Rab7, or Lamp1 expression plasmids were transfected into $\mathrm{CHO}$ or MDCK cells. Cells were fixed in $4 \%$ PFA after $48 \mathrm{~h}$ culturing and subjected to immunofluorescent staining. Primary antibodies: HA (Roche, 1:500); Flag (Sigma, 1:2 000); and Calreticulin (Abcam, 1:500). The CK1 inhibitor D4476 $(100 \mu \mathrm{M})$, Clathrin inhibitor CPZ (chlorpromazine, $30 \mu \mathrm{M})$, or Caveolin inhibitor Nystatin $(25 \mathrm{~g} / \mathrm{mL})$ was incubated with cells for 2-6 h prior to fixation.

\section{Quantitative PCR}

Total RNA was extracted from mouse embryos using the Trizol reagent (Invitrogen) and reverse transcribed using the Super- 
Script First-Strand Synthesis-System (Invitrogen). Quantitative PCR using SYBR Select Master Mix (Applied Biosystems) was performed on 7500 Fast Real-Time PCR system (Applied Biosystems). Total Vangl2 was detected using the following primers: 5'-GGAGTCGTGGAGATAAATCAGTG-3' and 5'-GACACTGTCCTCCATGTCCTTG-3' and Induced Vang12 was detected by: 5'-GGACTATGCAGGATCCTATCCA-3' and 5'-CGTCACTGATTTATCTCCACGA-3'. GAPDH mRNA level was used as internal control.

\section{Protein stability assay}

Mouse embryonic fibroblasts (MEFs) that stably express wild type and phospho-mutant HA-Vang12 were isolated from E12.5 $\beta$-actin-Cre;Rosa-Vangl2 $2^{W t /+}$ and $\beta$-actin-Cre;Rosa-Vangl2 ${ }^{A /+} \mathrm{em}$ bryos, respectively. To determine the stability of wild type and phospho-mutant Vangl2 proteins, these MEFs were treated with cycloheximide $(20 \mathrm{~g} / \mathrm{mL})$. Cells were harvested at different time points ( $0 \mathrm{~h}, 4 \mathrm{~h}, 8 \mathrm{~h}$, and $12 \mathrm{~h})$, lysed, and subjected to immunoblot analysis.

\section{Immunofluorescence and confocal microscopy}

For immunofluorescent staining of limb chondrocytes, E13.5 embryos were fixed in $4 \%$ paraformaldehyde/PBS at $4{ }^{\circ} \mathrm{C}$ for 30 min and incubated in $30 \%$ sucrose/PBS overnight. Embryonic limbs were embedded in OCT compound and $16 \mu \mathrm{m}$ sections were cut. Sections were permeabilized with $0.5 \%$ Triton X-100 in PBS for $5 \mathrm{~min}$, blocked with $3 \%$ bovine serum albumin in $0.1 \%$ Triton $\mathrm{X}-100 / \mathrm{PBS}$ for $1 \mathrm{~h}$, and incubated with primary antibodies overnight at $4{ }^{\circ} \mathrm{C}$. Primary antibodies against the following proteins and their dilutions were: $\beta$-catenin (BD Transduction, 1:500); HA (Roche, 1:500); Vang12 (SantaCruz, N13, 1:100); Lamp1 (DSHB, 1:500); and Rab5 (Abcam, 1:500). Secondary antibodies coupled to Alexa Fluor 488 or 568 of donkey anti-mouse, rat, or goat (Invitrogen) were diluted at 1:500 and incubated at room temperature for $1 \mathrm{~h}$. Confocal images were acquired by a Zeiss LSM710 microscope. Excitation wavelengths of 488 and $568 \mathrm{~nm}$ were used for the detection of secondary antibodies coupled with Alexa Fluor 488 and 568, respectively. Projected Z-stack images were acquired at $0.3 \mu \mathrm{m}$ intervals for $\sim 6 \mu \mathrm{m}$ and projected by Zeiss Zen software.

\section{Quantification of immunofluorescent intensity}

Immunofluorescent images of cells transfected with Vang12, Vang11, or Ror2 were analyzed by Image J software (NIH, v1.50i). Intensities of the total cell and intracellular areas were respectively measured. Intensities of three different areas around the cell were measured as background signal for calibration. The proportion of the membrane intensity (subtract intracellular intensity from total cell intensity) and intracellular intensity in total cell was calculated.

\section{Whole-mount immunostaining of cochlea and skin}

E18.5 embryonic heads (for cochlea) and E14.5 embryos (for skin) were fixed in $4 \%$ paraformaldehyde/PBS at $4{ }^{\circ} \mathrm{C}$ overnight and $1 \mathrm{~h}$, respectively. The inner ear cochlea and back skin were permeabilized with $0.5 \%$ Triton X-100 in PBS for 10 min and then blocked with $3 \%$ bovine serum albumin in $0.1 \%$ Triton X-100/ PBS for $1 \mathrm{~h}$. Primary antibodies including anti-acetylated-tubulin (Sigma, 1:2 000), anti- $\beta$-catenin (BD Transduction, 1:500), anti-HA (Roche, 1:500), anti-Lamp1 (DSHB, 1:500), or anti-Rab5 (Abcam, 1:500) were incubated overnight at $4{ }^{\circ} \mathrm{C}$. Secondary anti- bodies coupled to Alexa Fluor 488 and 568 of donkey anti-mouse and rat (1:500, Invitrogen) were incubated at room temperature for $1 \mathrm{~h}$. For cochlea staining, Alexa Fluor-488-coupled phalloidin (Invitrogen, 1:200) was incubated with cochlea for $15 \mathrm{~min}$ at room temperature. The actin-based stereocilium bundles were visualized by phalloidin staining, whereas the microtubule-based cilia were shown by staining with the acetylated-tubulin. Cochlea and skin were flat mounted. Confocal images were acquired with a Zeiss LSM710 confocal microscope. In the inner ear, all images were taken from the basal part of the cochlea.

\section{Quantification of cell shape and orientation}

Immunofluorescent images of limb chondrocytes and skin epidermal cells with $\beta$-catenin staining (cell membrane) were processed by CellProfiler 2.1.1, software developed by the Broad Institute to quantitatively measure cell phenotypes. CellProfiler automatically identifies objects in terms of the custom-designed pipeline and preset parameters (e.g., the range of the object size, the intensity threshold). The shape of the cell is reflected by the ratio of Major-Axis-Length to Minor-Axis-Length of the cell. The orientation of the cell is determined by the angle of the cells (in degrees ranging from $-90^{\circ}$ to $90^{\circ}$ ) between the $\mathrm{x}$-axis and the major axis of the cell. The tissue axis set for measurement is defined as shown in Supplementary information, Figure S5. Data were analyzed and plotted using Prism 6 (GraphPad) and Origin 9 software.

\section{Skeletal preparation}

E18.5 embryos were fixed for $\sim 2-3$ days in $100 \%$ ethanol and then transferred into $100 \%$ acetone after evisceration, followed by staining with Alizarin Red S (bone) and Alcian Blue (cartilage). Embryos were cleared by $1 \% \mathrm{KOH}$ and stored and photographed in $80 \%$ glycerol.

\section{Limb culturing}

The E11.5 embryonic forelimbs were dissected in pre-warmed DMEM containing 10\% FBS, pen/strep, and $20 \mathrm{mM}$ HEPES and cultured in pre-warmed 1:1 mixed DMEM (10\% FBS, pen/strep) and freshly prepared rat serum on a rotator at $37{ }^{\circ} \mathrm{C}$ with $5 \% \mathrm{CO}_{2}$ for 2 days. The culture medium and serum were changed every $24 \mathrm{~h}$. This culture method allows digit morphogenesis comparable to in vivo digit development from E11.5 to E12.5, a critical time window for initiation and establishment of PCP. CK1 inhibitor (20 $\mu \mathrm{M}$ or $1 \mu \mathrm{M}$ D4476, Abcam) was directly added into the culture medium and changed every $24 \mathrm{~h}$.

\section{Vangl2 phosphorylation assay by immunoblotting}

To detect the in vivo Vang12 phosphorylation, the embryonic tissues were lysed in lysis buffer (20 mM Tris- $\mathrm{HCl}(\mathrm{pH} 7.4), 150$ $\mathrm{mM} \mathrm{NaCl}, 1 \%$ Nonidet P-40) containing protease and phosphatase inhibitors. Proteins in the lysates were separated by electrophoresis on a $10 \%$ Bis-Tris Polyacrylamide Gel for Western blotting with anti-Vang12 antibody (Santa Cruz, N13, 1:200) or home-made phospho-specific Vangl2 antibodies (p-S84 or p-S82/S84). Vangl2, Ror 2, Dvl, CK1, or Wnt5a expression plasmids were introduced into cultured CHO, HEK293T, or MEF cells by transfection using Lipofectamine 3000 (Invitrogen). Cells were lysed after $48 \mathrm{~h}$ and subjected to immunoblot analysis. Primary antibodies were against the following proteins at the indicated dilutions: HA 
(Roche, 1:2 000); Flag (Sigma, 1:5 000); Myc (Santa Cruz, 1:1 000); and actin and tubulin (Sigma, 1:10 000). The CK1 inhibitor D4476 was dissolved in DMSO $(100 \mu \mathrm{M})$ and incubated with cells for $6 \mathrm{~h}$ prior to lysing cells. Wnt5a recombinant proteins were purchased from R\&D Systems and added into the medium for two hours (500 ng/mL).

\section{siRNA knockdown and co-immunoprecipitation}

Human $C K 1 \varepsilon / \delta$ siRNA (Ambion, Part\# 4390824 and 4392420), Dvl2/3 siRNA (synthesized at GenePharma), or negative control siRNA (GenePharma) were transfected into HEK293T cells using Lipofectamine RNAiMAX (Invitrogen) following the instructions of the manufacturer. $D v l 2$ and $D v l 3$ siRNA targeting sequences were described previously [110]. Dvl2: TCCACAATGTCTCTCAATA; $D v l 3$ : TAAACGAGATCAACTTTGA. The reverse transfection strategy was used and other plasmids were transfected 24 $\mathrm{h}$ after siRNA transfection. The cells were lysed $72 \mathrm{~h}$ after siRNA transfection. Antibodies were against the following endogenous proteins and used at the indicated dilutions: CK1 $1 \varepsilon$ and CK1 $\delta$ (Santa Cruz, 1:500) and Dvl2 and Dvl3 (Cell Signaling, 1:1 000). In the Co-IP experiment, transfected HEK293T cells were lysed in lysis buffer (20 mM Tris-HCl (pH 7.4), $150 \mathrm{mM} \mathrm{NaCl,} \mathrm{0.5 \%} \mathrm{Nonidet}$ $\mathrm{P}-40$ ) with protease and phosphatase inhibitors and pre-cleared by Protein A/G agarose (Santa Cruz). The cell lysates were then incubated with the anti-HA antibody (Roche, $0.5 \mu \mathrm{g}$ ) overnight at $4{ }^{\circ} \mathrm{C}$ followed by $2 \mathrm{~h}$ with Protein $\mathrm{A} / \mathrm{G}$ agarose (Santa Cruz). The immunoprecipitates were washed three times in lysis buffer, dissolved in sample buffer, and subjected to SDS-polyacrylamide electrophoresis and immunoblot analysis.

\section{Acknowledgments}

We thank Jun Cheng, Cecilia Rivas, and Elsa Escobar for their assistance in generating transgenic mice, Guadalupe Lopez for mouse husbandry and Jing Guo for confocal microscopy. The Ror 2 mutant mouse was provided by Yasuhiro Minami. The $\beta$-actin-Cre mouse was provided by Kathryn SE Cheah. The work in the Gao laboratory was supported by the University of Hong Kong Start-up funds and Hong Kong Research Grants Council (27115317). The work in the Yang laboratory was supported by the intramural research program of NHGRI at the US National Institutes of Health (NIH), NIH/NIAMS grant R01AR070877 and the Harvard School of Dental Medicine Start-up funds.

\section{Author Contributions}

YY and BG conceived and supervised the project. LG, GE and BG generated the transgenic mice. WY, DF and BG, assisted by XL, NW, YMW and NTC, performed the experiments. WY, YY and $\mathrm{BG}$ wrote the manuscript.

\section{Competing Financial Interests}

The authors declare no competing financial interests.

\section{References}

1 Wang Y, Nathans J. Tissue/planar cell polarity in vertebrates: new insights and new questions. Development 2007; 134:647-658.
2 Gray RS, Roszko I, Solnica-Krezel L. Planar cell polarity: coordinating morphogenetic cell behaviors with embryonic polarity. Dev Cell 2011; 21:120-133.

3 Devenport D. The cell biology of planar cell polarity. J Cell Biol 2014; 207:171-179.

4 Yang Y, Mlodzik M. Wnt-Frizzled/planar cell polarity signaling: cellular orientation by facing the wind (Wnt). Annu Rev Cell Dev Biol 2015; 31:623-646.

5 Butler MT, Wallingford JB. Planar cell polarity in development and disease. Nat Rev Mol Cell Biol 2017; 18:375-388.

6 Tree DR, Ma D, Axelrod JD. A three-tiered mechanism for regulation of planar cell polarity. Semin Cell Dev Biol 2002; 13:217-224.

7 Seifert JR, Mlodzik M. Frizzled/PCP signalling: a conserved mechanism regulating cell polarity and directed motility. Nat Rev Genet 2007; 8:126-138.

8 Vladar EK, Antic D, Axelrod JD. Planar cell polarity signaling: the developing cell's compass. Cold Spring Harbor Persp Biol 2009; 1:a002964.

9 Vinson CR, Adler PN. Directional non-cell autonomy and the transmission of polarity information by the frizzled gene of Drosophila. Nature 1987; 329:549-551.

10 Theisen H, Purcell J, Bennett M, Kansagara D, Syed A, Marsh JL. Dishevelled is required during wingless signaling to establish both cell polarity and cell identity. Development 1994; 120:347-360.

11 Adler PN, Krasnow RE, Liu J. Tissue polarity points from cells that have higher Frizzled levels towards cells that have lower Frizzled levels. Curr Biol 1997; 7:940-949.

12 Taylor J, Abramova N, Charlton J, Adler PN. Van Gogh: a new Drosophila tissue polarity gene. Genetics 1998; 150:199-210.

13 Wolff T, Rubin GM. Strabismus, a novel gene that regulates tissue polarity and cell fate decisions in Drosophila. Development 1998; 125:1149-1159.

14 Chae J, Kim MJ, Goo JH, et al. The Drosophila tissue polarity gene starry night encodes a member of the protocadherin family. Development 1999; 126:5421-5429.

15 Gubb D, Green C, Huen D, et al. The balance between isoforms of the prickle LIM domain protein is critical for planar polarity in Drosophila imaginal discs. Genes Dev 1999; 13:2315-2327.

16 Usui T, Shima Y, Shimada Y, et al. Flamingo, a seven-pass transmembrane cadherin, regulates planar cell polarity under the control of Frizzled. Cell 1999; 98:585-595.

17 Feiguin F, Hannus M, Mlodzik M, Eaton S. The ankyrin repeat protein Diego mediates Frizzled-dependent planar polarization. Dev Cell 2001; 1:93-101.

18 Torban E, Patenaude AM, Leclerc S, et al. Genetic interaction between members of the Vangl family causes neural tube defects in mice. Proc Natl Acad Sci USA 2008; 105:34493454.

19 Song H, Hu J, Chen W, et al. Planar cell polarity breaks bilateral symmetry by controlling ciliary positioning. Nature 2010; 466:378-382.

20 Hamblet NS, Lijam N, Ruiz-Lozano P, et al. Dishevelled 2 is essential for cardiac outflow tract development, somite segmentation and neural tube closure. Development 2002; 129:5827-5838 
21 Wang J, Hamblet NS, Mark S, et al. Dishevelled genes mediate a conserved mammalian PCP pathway to regulate convergent extension during neurulation. Development 2006; 133:1767-1778.

22 Etheridge SL, Ray S, Li S, et al. Murine dishevelled 3 functions in redundant pathways with dishevelled 1 and 2 in normal cardiac outflow tract, cochlea, and neural tube development. PLoS Genet 2008; 4:e1000259.

23 Lu X, Borchers AG, Jolicoeur C, Rayburn H, Baker JC, Tessier-Lavigne M. PTK7/CCK-4 is a novel regulator of planar cell polarity in vertebrates. Nature 2004; 430:93-98.

24 Gao B, Song H, Bishop K, et al. Wnt signaling gradients establish planar cell polarity by inducing Vang12 phosphorylation through Ror2. Dev Cell 2011; 20:163-176.

25 Macheda ML, Sun WW, Kugathasan K, et al. The Wnt receptor Ryk plays a role in mammalian planar cell polarity signaling. J Biol Chem 2012; 287:29312-29323.

26 Andre P, Wang Q, Wang N, et al. The Wnt coreceptor Ryk regulates Wnt/planar cell polarity by modulating the degradation of the core planar cell polarity component Vang12. $J$ Biol Chem 2012; 287:44518-44525.

27 Zallen JA. Planar polarity and tissue morphogenesis. Cell 2007; 129:1051-1063.

28 Goodrich LV, Strutt D. Principles of planar polarity in animal development. Development 2011; 138:1877-1892.

29 Montcouquiol M, Rachel RA, Lanford PJ, Copeland NG, Jenkins NA, Kelley MW. Identification of Vang12 and Scrb1 as planar polarity genes in mammals. Nature 2003; 423:173177.

30 Hashimoto M, Shinohara K, Wang J, et al. Planar polarization of node cells determines the rotational axis of node cilia. Nat Cell Biol 2010; 12:170-176.

31 Caddy J, Wilanowski T, Darido C, et al. Epidermal wound repair is regulated by the planar cell polarity signaling pathway. Dev Cell 2010; 19:138-147.

32 Shafer B, Onishi K, Lo C, Colakoglu G, Zou Y. Vangl2 promotes Wnt/planar cell polarity-like signaling by antagonizing Dvl1-mediated feedback inhibition in growth cone guidance. Dev Cell 2011; 20:177-191.

33 Gao B, Yang Y. Planar cell polarity in vertebrate limb morphogenesis. Curr Opin Genet Dev 2013; 23:438-444.

34 Simons M, Mlodzik M. Planar cell polarity signaling: from fly development to human disease. Annu Rev Genet 2008; 42:517-540.

35 Wallingford JB, Niswander LA, Shaw GM, Finnell RH. The continuing challenge of understanding, preventing, and treating neural tube defects. Science 2013; 339:1222002.

36 De Marco P, Merello E, Piatelli G, Cama A, Kibar Z, Capra V. Planar cell polarity gene mutations contribute to the etiology of human neural tube defects in our population. Birth Defects Res A Clin Mol Teratol 2014; 100:633-641.

37 Tao H, Manak JR, Sowers L, et al. Mutations in prickle orthologs cause seizures in flies, mice, and humans. Am J Hum Genet 2011; 88:138-149.

38 Wang B, Sinha T, Jiao K, Serra R, Wang J. Disruption of PCP signaling causes limb morphogenesis and skeletal defects and may underlie Robinow syndrome and brachydactyly type B. Hum Mol Genet 2011; 20:271-285.

39 Hayes M, Gao X, Yu LX, et al. ptk7 mutant zebrafish models of congenital and idiopathic scoliosis implicate dysregulated Wnt signalling in disease. Nat Commun 2014; 5:4777.

40 Andersen MR, Farooq M, Koefoed K, et al. Mutation of the planar cell polarity gene VANGL1 in adolescent idiopathic scoliosis. Spine 2017; 42:E702-E707.

41 Luga V, Zhang L, Viloria-Petit AM, et al. Exosomes mediate stromal mobilization of autocrine Wnt-PCP signaling in breast cancer cell migration. Cell 2012; 151:1542-1556.

42 van Bokhoven $\mathrm{H}$, Celli J, Kayserili H, et al. Mutation of the gene encoding the ROR2 tyrosine kinase causes autosomal recessive Robinow syndrome. Nat Genet 2000; 25:423-426.

43 Afzal AR, Rajab A, Fenske CD, et al. Recessive Robinow syndrome, allelic to dominant brachydactyly type B, is caused by mutation of ROR2. Nat Genet 2000; 25:419-422.

44 Person AD, Beiraghi S, Sieben CM, et al. WNT5A mutations in patients with autosomal dominant Robinow syndrome. Dev Dyn 2010; 239:327-337.

45 White J, Mazzeu JF, Hoischen A, et al. DVL1 frameshift mutations clustering in the penultimate exon cause autosomal-dominant Robinow syndrome. Am J Hum Genet 2015; 96:612-622.

46 White JJ, Mazzeu JF, Hoischen A, et al. DVL3 alleles resulting in a -1 frameshift of the last exon mediate autosomal-dominant Robinow syndrome. Am J Hum Genet 2016; 98:553-561.

47 Axelrod JD. Unipolar membrane association of Dishevelled mediates Frizzled planar cell polarity signaling. Genes Dev 2001; 15:1182-1187.

48 Strutt DI. Asymmetric localization of frizzled and the establishment of cell polarity in the Drosophila wing. Mol Cell 2001; 7:367-375.

49 Lawrence PA, Casal J, Struhl G. Cell interactions and planar polarity in the abdominal epidermis of Drosophila. Development 2004; 131:4651-4664.

50 Chen WS, Antic D, Matis M, et al. Asymmetric homotypic interactions of the atypical cadherin flamingo mediate intercellular polarity signaling. Cell 2008; 133:1093-1105.

51 Wallingford JB. Planar cell polarity and the developmental control of cell behavior in vertebrate embryos. Annu Rev Cell Dev Biol 2012; 28:627-653.

52 Devenport D. Tissue morphodynamics: Translating planar polarity cues into polarized cell behaviors. Semin Cell Dev Biol 2016; 55:99-110.

53 Lawrence PA, Sanson B, Vincent JP. Compartments, wingless and engrailed: patterning the ventral epidermis of Drosophila embryos. Development 1996; 122:4095-4103.

54 Casal J, Struhl G, Lawrence PA. Developmental compartments and planar polarity in Drosophila. Curr Biol 2002; 12:1189-1198.

55 Lawrence PA, Struhl G, Casal J. Planar cell polarity: one or two pathways? NatRev Genet 2007; 8:555-563.

56 Aigouy B, Farhadifar R, Staple DB, et al. Cell flow reorients the axis of planar polarity in the wing epithelium of Drosophila. Cell 2010; 142:773-786.

57 Sagner A, Merkel M, Aigouy B, et al. Establishment of global patterns of planar polarity during growth of the Drosophila wing epithelium. Curr Biol 2012; 22:1296-1301.

58 Matis M, Axelrod JD. Regulation of PCP by the Fat signaling pathway. Genes Dev 2013; 27:2207-2220. 
59 Chien YH, Keller R, Kintner C, Shook DR. Mechanical strain determines the axis of planar polarity in ciliated epithelia. Curr Biol 2015; 25:2774-2784.

60 Aw WY, Heck BW, Joyce B, Devenport D. Transient tissue-scale deformation coordinates alignment of planar cell polarity junctions in the mammalian skin. Curr Biol 2016; 26:2090-2100.

61 Aw WY, Devenport D. Planar cell polarity: global inputs establishing cellular asymmetry. Curr Opin Cell Biol 2017; 44:110-116.

62 Parr BA, Shea MJ, Vassileva G, McMahon AP. Mouse Wnt genes exhibit discrete domains of expression in the early embryonic CNS and limb buds. Development 1993; 119:247261.

63 Fisher ME, Clelland AK, Bain A, et al. Integrating technologies for comparing 3D gene expression domains in the developing chick limb. Dev Biol 2008; 317:13-23.

64 Witte F, Dokas J, Neuendorf F, Mundlos S, Stricker S. Comprehensive expression analysis of all Wnt genes and their major secreted antagonists during mouse limb development and cartilage differentiation. Gene Expr Patterns 2009; 9:215-223.

65 Wu J, Roman AC, Carvajal-Gonzalez JM, Mlodzik M. Wg and Wnt4 provide long-range directional input to planar cell polarity orientation in Drosophila. Nat Cell Biol 2013; 15:1045-1055.

66 Chu CW, Sokol SY. Wnt proteins can direct planar cell polarity in vertebrate ectoderm. Elife 2016; 5.

67 Gros J1, Serralbo O, Marcelle C. WNT11 acts as a directional cue to organize the elongation of early muscle fibres. Nature 2009; 457:589-593.

68 Minegishi K, Hashimoto M, Ajima R, et al. A Wnt5 activity asymmetry and intercellular signaling via PCP proteins polarize node cells for left-right symmetry breaking. Dev Cell 2017; 40:439-452.e4.

69 Ossipova O, Kim K, Sokol SY. Planar polarization of Vang12 in the vertebrate neural plate is controlled by Wnt and Myosin II signaling. Biol Open 2015; 4:722-730.

70 Kelly LK, Wu J, Yanfeng WA, Mlodzik M. Frizzled-induced Van Gogh phosphorylation by CK1epsilon promotes asymmetric localization of core PCP factors in Drosophila. Cell Rep 2016; 16:344-356.

71 Kibar Z, Bosoi CM, Kooistra M, et al. Novel mutations in VANGL1 in neural tube defects. Hum Mut 2009; 30:E706-E715.

72 Lei YP, Zhang T, Li H, Wu BL, Jin L, Wang HY. VANGL2 mutations in human cranial neural-tube defects. Eng $J$ Med 2010; 362:2232-2235.

73 Hubner K, Windoffer R, Hutter H, Leube RE. Tetraspan vesicle membrane proteins: synthesis, subcellular localization, and functional properties. Int Rev Cytol 2002; 214:103-159.

74 Belotti E, Puvirajesinghe TM, Audebert S, et al. Molecular characterisation of endogenous Vang12/Vangl1 heteromeric protein complexes. PloS One 2012; 7:e46213.

75 Yin H, Copley CO, Goodrich LV, Deans MR. Comparison of phenotypes between different vangl2 mutants demonstrates dominant effects of the Looptail mutation during hair cell development. PLoS One 2012; 7:e31988.

76 Torban E, Wang HJ, Groulx N, Gros P. Independent muta- tions in mouse Vangl2 that cause neural tube defects in looptail mice impair interaction with members of the Dishevelled family. J Biol Chem 2004; 279:52703-52713.

77 Soriano P. Generalized lacZ expression with the ROSA26 Cre reporter strain. Nat Genet 1999; 21:70-71.

78 Lewandoski M, Meyers EN, Martin GR. Analysis of Fgf8 gene function in vertebrate development. Cold Spring Harb Symp Quant Biol 1997; 62:159-168.

79 Hayashi S, Lewis P, Pevny L, McMahon AP. Efficient gene modulation in mouse epiblast using a Sox2Cre transgenic mouse strain. Gene Expr Patterns 2002; 2:93-97.

80 Gravel M, Iliescu A, Horth C, Apuzzo S, Gros P. Molecular and cellular mechanisms underlying neural tube defects in the loop-tail mutant mouse. Biochemistry 2010; 49:34453455 .

81 Devenport D, Fuchs E. Planar polarization in embryonic epidermis orchestrates global asymmetric morphogenesis of hair follicles. Nat Cell Biol 2008; 10:1257-1268.

82 Strong LC, Hollander WF. Hereditary loop-tail in the house mouse. J Hered 1949; 40:329-334.

83 Kibar Z, Vogan KJ, Groulx N, Justice MJ, Underhill DA, Gros P. Ltap, a mammalian homolog of Drosophila Strabismus/Van Gogh, is altered in the mouse neural tube mutant Loop-tail. Nat Genet 2001; 28:251-255.

84 Curtin JA, Quint E, Tsipouri V, et al. Mutation of Celsr1 disrupts planar polarity of inner ear hair cells and causes severe neural tube defects in the mouse. Curr Biol 2003; 13:11291133.

85 Rena G, Bain J, Elliott M, Cohen P. D4476, a cell-permeant inhibitor of CK1, suppresses the site-specific phosphorylation and nuclear exclusion of FOXO1a. EMBO Rep 2004; 5:60-65.

86 Strutt H, Price MA, Strutt D. Planar polarity is positively regulated by casein kinase Iepsilon in Drosophila. Curr Biol 2006; 16:1329-1336.

87 Klein TJ, Jenny A, Djiane A, Mlodzik M. CKIepsilon/discs overgrown promotes both Wnt-Fz/beta-catenin and Fz/PCP signaling in Drosophila. Curr Biol 2006; 16:1337-1343.

88 Peters JM, McKay RM, McKay JP, Graff JM. Casein kinase I transduces Wnt signals. Nature 1999; 401:345-350.

89 Yanfeng WA, Berhane H, Mola M, Singh J, Jenny A, Mlodzik M. Functional dissection of phosphorylation of Disheveled in Drosophila. Dev Biol 2011; 360:132-142.

90 Jiang J. CK1 in developmental signaling: Hedgehog and Wnt. Curr Top Dev Biol 2017; 123:303-329.

91 Price MA. CKI, there's more than one: casein kinase I family members in Wnt and Hedgehog signaling. Genes Dev 2006; 20:399-410.

92 Etchegaray JP, Machida KK, Noton E, et al. Casein kinase 1 delta regulates the pace of the mammalian circadian clock. Mol Cell Biol 2009; 29:3853-3866.

93 Gao C, Chen YG. Dishevelled: The hub of Wnt signaling. Cell Signal 2010; 22:717-727.

94 Axelrod JD. Progress and challenges in understanding planar cell polarity signaling. Semin Cell Dev Biol 2009; 20:964971.

95 Tree DR, Shulman JM, Rousset R, Scott MP, Gubb D, Axelrod JD. Prickle mediates feedback amplification to generate asymmetric planar cell polarity signaling. Cell 2002; 
109:371-381.

96 Amonlirdviman K, Khare NA, Tree DR, Chen WS, Axelrod JD, Tomlin CJ. Mathematical modeling of planar cell polarity to understand domineering nonautonomy. Science 2005; 307:423-426.

97 Chen W, ten Berge D, Brown J, et al. Dishevelled 2 recruits beta-arrestin 2 to mediate Wnt5A-stimulated endocytosis of Frizzled 4. Science 2003; 301:1391-1394.

98 Wu J, Mlodzik M. The frizzled extracellular domain is a ligand for Van Gogh/Stbm during nonautonomous planar cell polarity signaling. Dev Cell 2008; 15:462-469.

99 Martinez S, Scerbo P, Giordano M, et al. The PTK7 and ROR2 protein receptors interact in the vertebrate WNT/ planar cell polarity (PCP) pathway. J Biol Chem 2015; 290:30562-30572.

100 Witte F, Bernatik O, Kirchner K, et al. Negative regulation of Wnt signaling mediated by CK1-phosphorylated Dishevelled via Ror2. FASEB J 2010; 24:2417-2426.

101 Grumolato L, Liu G, Mong P, et al. Canonical and noncanonical Wnts use a common mechanism to activate completely unrelated coreceptors. Genes Dev 2010; 24:2517-2530.

102 Axelrod JD, Miller JR, Shulman JM, Moon RT, Perrimon N. Differential recruitment of Dishevelled provides signaling specificity in the planar cell polarity and Wingless signaling pathways. Genes Dev 1998; 12:2610-2622.

103 Shimada Y, Yonemura S, Ohkura H, Strutt D, Uemura T. Polarized transport of Frizzled along the planar microtubule ar- rays in Drosophila wing epithelium. Dev Cell 2006; 10:209222.

104 Yu A, Rual JF, Tamai K, et al. Association of Dishevelled with the clathrin AP-2 adaptor is required for Frizzled endocytosis and planar cell polarity signaling. Dev Cell 2007; 12:129-141.

105 Strutt H, Strutt D. Differential stability of flamingo protein complexes underlies the establishment of planar polarity. Curr Biol 2008; 18:1555-1564.

106 Wansleeben C, Feitsma H, Montcouquiol M, Kroon C, Cuppen E, Meijlink F. Planar cell polarity defects and defective Vang12 trafficking in mutants for the COPII gene Sec $24 b$. Development 2010; 137:1067-1073.

107 Devenport D, Oristian D, Heller E, Fuchs E. Mitotic internalization of planar cell polarity proteins preserves tissue polarity. Nat Cell Biol 2011; 13:893-902.

108 Ossipova O, Kim K, Lake BB, Itoh K, Ioannou A, Sokol SY. Role of Rab11 in planar cell polarity and apical constriction during vertebrate neural tube closure. Nat Commun 2014; 5:3734.

109 Cho B, Pierre-Louis G, Sagner A, Eaton S, Axelrod JD. Clustering and negative feedback by endocytosis in planar cell polarity signaling is modulated by ubiquitinylation of prickle. PLoS Genet 2015; 11:e1005259.

110 Pan W, Choi SC, Wang H, et al. Wnt3a-mediated formation of phosphatidylinositol 4,5-bisphosphate regulates LRP6 phosphorylation. Science 2008; 321:1350-1353.

(Supplementary information is linked to the online version of the paper on the Cell Research website.) 